Preprint typeset in JHEP style - HYPER VERSION

DAMTP-2003-146

\title{
Quantum Mechanics of Yano tensors: Dirac equation in curved spacetime
}

\author{
Marco Cariglia \\ DAMTP, Centre for Mathematical Sciences, Cambridge University \\ Wilberforce Road, Cambridge CB3 OWA, UK \\ E-mail: M.Cariglia@damtp.cam.ac.uk
}

\begin{abstract}
In spacetimes admitting Yano tensors the classical theory of the spinning particle possesses enhanced worldline supersymmetry. Quantum mechanically generators of extra supersymmetries correspond to operators that in the classical limit commute with the Dirac operator and generate conserved quantities. We show that the result is preserved in the full quantum theory, that is, Yano symmetries are not anomalous. This was known for Yano tensors of rank two, but our main result is to show that it extends to Yano tensors of arbitrary rank. We also describe the conformal Yano equation and show that is invariant under Hodge duality. There is a natural relationship between Yano tensors and supergravity theories. As the simplest possible example, we show that when the spacetime admits a Killing spinor then this generates Yano and conformal Yano tensors. As an application, we construct Yano tensors on maximally symmetric spaces: they are spanned by tensor products of Killing vectors.
\end{abstract}

KEYwords: Dirac equation, Yano operators, supersymmetry, special holonomy. 


\section{Contents}

1. Introduction. 1

2. The spinning particle 1

2.1 The classical theory 5

2.2 Supersymmetry and Yano tensors

2.3 The quantum system 9

2.4 Yano operators 10

3. Conformal Yano equation and Hodge duality 12

4. Spaces of almost special holonomy 13

5. Maximally symmetric spaces 17

5.1 Geometry of symmetric spaces 17

5.2 Yano tensors on maximally symmetric spaces $\overline{19}$

6. Conclusions 24

Appendix 24

\section{Introduction.}

The concept of symmetry, and especially isometry, has been extensively used in General Relativity in the past. However, already in 1979 Collins [1] pointed out that the ordinary use of symmetries had reached a "stagnation point", and few further results could be obtained along the same direction. Symmetries of a Hamiltonian system in curved spacetimes are in one to one correspondence to Killing tensors. These are symmetric tensors $K^{\mu_{1} \ldots \mu_{n}}=K^{\left(\mu_{1} \ldots \mu_{n}\right)}$, associated to conserved quantities of degree $n$ in the momentum variables, and generate symplectic transformations in the phase space of the system. These transformations are determined by a vector flow. Ordinary Killing vectors generate isometries and correspond to the pushforward on coordinate space of the vector flow. Higher rank Killing tensors instead, which are defined by the condition $D_{(\lambda} K_{\left.\mu_{1} \ldots \mu_{n}\right)}=0$, have zero pushforward: they correspond to genuine symmetries of the whole phase space, and not only of configuration space.

The next simple object that can be studied on a manifold $M$ after Killing tensors is a Yano tensor. Such objects were introduced from a purely mathematical point of view in 1951 by Yano [2]. The physical interpretation has remained obscure until Floyd [3] 
and Penrose [4] showed that the Stackel-Killing tensor $K^{\mu \nu}$ of the four-dimensional KerrNewman spacetime admits a square root, that is an antisymmetric tensor $f^{\mu \nu}=f^{[\mu \nu]}$ such that

$$
K^{\mu \nu}=f^{\mu \lambda} f_{\lambda}^{\nu}
$$

$f^{\mu \nu}$ proves indeed to be a Yano tensor and Carter and McLenaghan [5] were then able to construct from it a linear differential operator that commutes with the Dirac operator. This provides a further quantum number for the spinor wavefunction and explains why separation of variables can be achieved for the Dirac equation on that background. Gibbons, Rietdijk and van Holten [6] gave a systematic description of the role of rank 2 Yano tensors in General Relativity. They generate additional supercharges in the dynamics of pseudo-classical spinning particles moving in $M$. The Yano condition ensures that these supercharges are superinvariant. They generate a classical superalgebra (in the sense of Poisson-Dirac brackets) that closes on conserved quantities associated to Killing tensors of rank 2. Tanimoto [7] generalized the construction to Yano tensors of generic rank $p$, which always generate rank 2 Killing tensors when squared. Yano tensors generated by the action of groups on a spacetime have been discussed for example in [8], [9].

Two ingredients of the construction outlined above are physically appealing and make the further study of Yano tensors a worthwhile task.

The first is the connection between Yano tensors and supersymmetry, both at the classical and quantum level. Yano tensors are paired to their corresponding Killing tensors (hence in some loose sense "superpartners"), in the same way that the Dirac operator is paired to its square, the Hamiltonian $H$. They also allow us to consider at the same level the equation of motion for scalar and spinor wave functions: on one side there is the CarterMcLenaghan like operator which commutes with the Dirac operator and hence with the spinorial Hamiltonian. On the other side, from the square of the Yano tensor one can construct an operator acting on scalars of the form $K=K^{\mu \nu} D_{\mu} D_{\nu}$, where $K^{\mu \nu}$ is given by (1.1) and $D_{\mu}$ is the covariant derivative on $M$. Then $K$ automatically commutes with the scalar Laplacian $\Delta=D_{\mu} g^{\mu \nu} D_{\nu}$. This is quite unexpected since in general the operator $K$ does not define a genuine quantum mechanical symmetry. The reason is that on a generic curved spacetime $M$ there appears a quantum anomaly proportional to a contraction of $K^{\mu \nu}$ with the Ricci tensor. However, when the Killing tensor $K^{\mu \nu}$ is of the form (1.1), then the anomaly disappears thanks to an integrability condition satisfied by the Yano tensor. Most important of all, Yano tensors generate an exotic superalgebra, which is more general than an usual extended superalgebra.

The second ingredient is the fact that Yano tensors, with their non standard superalgebra, allow us to consider extended supersymmetry in manifolds more general than Kähler. Usually one considers String Theory, which can be thought of as a two-dimensional supersymmetric sigma model, where demanding to have extended supersymmetry on the worldsheet always leads to a Kähler structure in target space. Instead for the spinning particle, which is a one dimensional supersymmetric sigma model, having a smaller worldsheet implies a greater set of possible target spacetimes, that are all those which admit Yano tensors. Therefore, Yano tensors are of direct interest in the study of manifolds that 
are less restricted than Kähler ones.

In this paper we begin a systematic study of Yano tensors and their relation with Quantum Mechanics. The results quoted so far about Yano tensors are purely classical, that is the supercharges associated to them generate extra supersymmetries at the level of Poisson-Dirac brackets. Only in the case of rank 2 Yano tensors the result of Carter and McLenaghan is quantum mechanical. In principle, when quantizing the theory of the spinning particle, quantum mechanical anomalies could appear in the commutation relations due to the fact that spacetime is not flat. The first step in understanding the role of Yano tensors then becomes that of analysing what happens at the quantum mechanical level. What we discover and present here as the main result obtained is that indeed anomalies are completely absent, independently of the rank of the Yano tensor considered. The result is quite remarkable since in principle there is no particular reason why the conservation laws should be quantum mechanically protected.

There seems to be a relationship between the absence of anomalies and the fact that Yano tensors are completely antisymmetric objects. Ordinary conservation laws associated to symmetric tensors, like the energy momentum tensor or Killing tensors, are usually plagued by quantum mechanical anomalies. What emerges here instead is that for antisymmetric objects, which are somehow more fundamental, there are no anomalies. Nevertheless, we still lack a clear geometrical understanding of why this happens.

The main features of the system remind the dynamics of $p$-forms on a compact Riemannian manifold $V$. The bundle of differential forms $\Lambda^{*}(V)$ over $V$ can be decomposed into the bundle of even and odd forms. Then the operator $d+\delta$, where $d$ is the exterior derivative and $\delta$ its adjoint, is elliptic and defines the de Rham complex on $V$. Supersymmetry can be implemented by defining a real (Majorana) supercharge to be $Q=d+\delta: \Lambda^{\text {even }}(V) \rightarrow$ $\Lambda^{\text {odd }}(V)$. Then $Q^{\dagger}=d+\delta: \Lambda^{\text {odd }}(V) \rightarrow \Lambda^{\text {even }}(V)$ and the superalgebra is simply $\left\{Q, Q^{\dagger}\right\}=$ $2 \Delta:=2 H$. Energy is bounded from below by zero. 'Bosonic' and 'fermionic' degrees of freedom at the same at energy level $E \neq 0$ are paired, since $Q$ is an elliptic operator. However, in order to see if supersymmetry is preserved one has to consider the Witten index, which coincides with the index of the Laplacian. It is well known that the statement about the index is purely topological, it does not depend on the metric on $V$, and the reason is that one is dealing with antisymmetric objects, the $p$-forms. Therefore, once an appropriate manifold $V$ is chosen, supersymmetry will be present with no anomalies, independently of the metric one puts on it. This is directly analogous to what happens with Yano operators: in that case, the only possible anomalies that could appear are proportional to the Riemann tensor. Their absence can in principle mean that the underlying structure is metric independent.

It then seems evident that Yano operators play an important role in quantum mechanics. They provide exactly conserved quantum numbers for the Dirac equation and might be relevant in the study of the scalar Laplace operator on $M$ and of its higher spin (both integer and semi integer) counterparts, due to a profound geometrical meaning which still needs to be uncovered. One possible geometrical setting where Yano tensors and their related operators can acquire a proper meaning is that of complexes and index theorems. Yano operators can be considered as exotic generalizations of the Dirac operator $\not D$. In some cases 
their square defines a (pseudo) elliptic operator with an associated index. Therefore they generate a new kind of complex which is very special, since they commute/anticommute with the Dirac operator. In [10] it has been shown that as a consequence Yano operators and Dirac operators have the same index. In [11] one example of this correspondence, involving index theorems with torsion on Taub-NUT spaces, has been explicitly worked out.

Motivated by these considerations, we look for spacetimes admitting Yano tensors. It is easy to show that this is true for all spacetimes of almost special holonomy. Such spacetimes are of interest both from the mathematical point of view, because they are a rather general class of manifolds, and from the physical point of view, since they can arise as solutions for compactifications of supergravity theories in higher dimensions that preserve some supersymmetry in presence of fluxes. For example one could use the new conserved quantities to solve Dirac equation on singular (almost) $G_{2}$ spacetimes, and look for chiral fermions. Main feature is that almost special holonomy spaces admit Killing spinors. Given a Killing spinor, it generates a tower of antisymmetric tensors of rank $1 \leq p \leq D=\operatorname{dim}(M)$, which we show to be either Yano or conformal Yano, depending whether $p$ is even or odd. Conformal Yano tensors are a generalization of Yano tensors. The set of conformal Yano tensors is invariant under the Hodge duality operation and Yano tensors are in correspondence with closed conformal Yano tensors.

As a concrete example we realize this construction on maximally symmetric spaces, all those that locally are given by spheres, hyperbolic spaces, de Sitter and Anti-de Sitter. On such spaces it is easy to solve the Killing equation explicitly and in a manifestly coordinate independent way. Hence we show that Yano and conformal Yano tensors on these spaces are extremely simple in form and given by a sum of products of Killing vectors.

The rest of the paper is organized as follows. A substantial part of it is dedicated to a review of background material of various kind. We begin with an extensive review of the theory of the classical spinning particle and its quantization in 2. The reader who is already acquainted with it can skip it altogether and read only sec.2.4, where quantum Yano operators are introduced. There we discuss the absence of anomalies. In 3 we derive the conformal Yano equation and describe its relationship with the usual Yano equation and Hodge duality. This turns out to be useful in the following 4 , where we review some basic notions and then show that spaces of almost special holonomy host a tower of antisymmetric tensors which are either Yano tensors or conformal Yano. As a concrete application, we explicitly construct such tensors on maximally symmetric spacetimes. In 5.1 we present well known results about the geometry of symmetric spaces and apply them in 5.2 to the solution of the Killing equation when there is maximal symmetry. From Killing spinors we construct Yano tensors and show that on these spacetimes they are always given by a sum of tensor products of Killing vectors. We end in section 6 with a summary and concluding remarks.

\section{The spinning particle}

Here we describe the theory of the classical spinning particle in curved spacetime and its 
quantization. We show that Yano operators commute with the Dirac operator and therefore the quantum theory admits genuine extra supersymmetries.

\subsection{The classical theory}

In this section we briefly summarize the classical theory of a spinning particle in curved spacetime. Even if it is well known, it is the natural setting where Yano tensors arise and acquire their physical meaning. In the following, greek indices $\mu, \nu, \ldots$ represent tensor components in arbitrary reference frames while latin ones $a, b, \ldots$ are referred to locally Lorentzian frames. Conversion between the two is given by using the vielbein $e_{\mu}^{a}(x)$.

Let $M$ be a spacetime. The spinning particle can be obtained as a supersymmetric formulation of the ordinary point particle in $M$, and is known to be the pseudo-classical limit of Dirac's theory of spin 1/2 fermions [12, 13, 14, 15, 16, 17].

The configuration space of the theory is given by a bundle which is a fermionic extension of the tangent bundle $T(M)$. Fermionic coordinates are special in that they behave at the same time as coordinates and momenta, as is well known. Let $x^{\mu}, \mu=1, \ldots, D$, where $D=\operatorname{dim}(M)$, be bosonic coordinates for $M$. First of all one has to define a fermionic extension of $M$ itself. Take $M_{\psi}$ to be the bundle with base $M$, described locally by the coordinates $\left(x^{\mu}, \psi^{\nu}\right)$, where the $\psi^{\nu}$ are Grassmannian variables. Under a change of variables in the base manifold $M$ the Grassmannian coordinates $\psi^{\nu}$ have the same transition functions as a vector in the tangent space of $M, T(M)$, and the bundle is well defined. $M_{\psi}$ is the configuration space. A particle with spin moving in the spacetime is described by a curve $\tau \mapsto(x(\tau), \psi(\tau)) \in M_{\psi}, \tau \in \mathbb{R}$. The Grassmann vector has the physical interpretation of being the spin of a particle, more on this will be found below in this section. One needs to define a connection on $M_{\psi}$, and the most natural choice is to use the same connection of $T(M)$ :

$$
\nabla_{\mu} \psi^{\nu}=\partial_{\mu} \psi^{\nu}+\Gamma_{\mu \rho}^{\nu} \psi^{\rho}
$$

The curve describing the particle on $M_{\psi}$ can be obtained by a lift of the curve $\tau \mapsto x(\tau)$ by asking that $\psi^{\nu}$ is parallely transported:

$$
\frac{D \psi^{\nu}}{D t}:=\dot{x}^{\mu} \nabla_{\mu} \psi^{\nu}=0
$$

Geometrically this corresponds to splitting the tangent bundle of $M_{\psi}, T\left(M_{\psi}\right)$, into a vertical subbundle $T^{v}\left(M_{\psi}\right)$, with fibre generated by the vectors $\left\{\partial / \partial \psi^{\nu}\right\}$, and a horizontal subbundle $T^{h}\left(M_{\psi}\right)$, with fibre generated by the vectors

$$
D_{\mu}:=\partial_{\mu}-\Gamma_{\mu \nu}^{\lambda} \psi^{\nu} \frac{\partial}{\partial \psi^{\lambda}}
$$

Then, along the lifted curve, for every function $f=f(x, \psi)$ on $M_{\psi}$ one has

$$
\frac{d f}{d t}=\dot{x}^{\mu} \frac{\partial f}{\partial x^{\mu}}+\dot{\psi}^{\nu} \frac{\partial f}{\partial \psi^{\nu}}=\dot{x}^{\mu} D_{\mu}(f) .
$$


This means, as is well known, that $T^{h}\left(M_{\psi}\right)$ is isomorphic to $T(M)$. Now it becomes easy to describe the lift $\tau \mapsto(x(\tau))$ to the whole fermionic tangent bundle $T\left(M_{\psi}\right)$ : first lifting it to $M_{\psi}$ using eq. 2.2), and then one choosing a connection on $T^{h}\left(M_{\psi}\right)$, defined by

$$
\nabla_{\mu}^{h} v^{\nu}:=\partial_{\mu} v^{\nu}+\Gamma_{\mu \lambda}^{\nu} v^{\lambda}
$$

We then ask for the lift of the curve to satisfy the equation

$$
\frac{D^{2} x^{\mu}}{D \tau^{2}}:=\dot{x}^{\lambda} \nabla_{\lambda}^{h} \dot{x}^{\mu}=\frac{1}{2} R_{\nu}^{\mu} \dot{x}^{\nu},
$$

where we have defined $R_{\mu \nu}:=i / 2 R_{\mu \nu \lambda_{1} \lambda_{2}} \psi^{\lambda_{1}} \psi^{\lambda_{2}}$. Eqs.(2.2), (2.6) define generalized geodesics on $M_{\psi}$. The term $R_{\nu}^{\mu} \dot{x}^{\nu}$ comes from physical requirements, basically from the supersymmetry of the Lagrangian. In fact, as in the bosonic case, there is a Lagrangian formulation that generates such curves. The Lagrangian density is nothing else than a supersymmetric extension of the Lagrangian density that gives geodesics on $M$. It is invariant under super-reparametrizations of $\tau$. A suitable gauge choice gives the following form for the Lagrangian density:

$$
L=\frac{1}{2} g_{\mu \nu} \dot{x}^{\mu} \dot{x}^{\nu}+\frac{1}{2} i g_{\mu \nu} \psi^{\mu} \frac{D \psi^{\nu}}{D \tau} .
$$

The gauge choice is expressed by two conditions: that the trajectory is null, and that spin is spacelike,

$$
\dot{x}^{\mu} \psi_{\mu}=0
$$

The equations of motion that come from (2.7) are exactly (2.2), (2.6). The particle is massless. No mass is admitted since in the original, super-reparametrization invariant Lagrangian it is not possible to construct a fermionic mass term. However, at the quantum level it will still be possible to impose a mass condition.

In order to move to the Hamiltonian formulation and quantize the system it is necessary to take into account that the momentum conjugate to $\psi$ is proportional to $\psi$ itself. This is a second class constraint that can be eliminated in a standard way and yields the PoissonDirac brackets

$$
\begin{gathered}
\left\{x^{\mu}, p_{\nu}\right\}=\delta_{\nu}^{\mu}, \\
\left\{\psi^{\mu}, \psi_{\nu}\right\}=-i \delta_{\nu}^{\mu} .
\end{gathered}
$$

However, $x^{\mu}, p_{\nu}$ and $\psi^{\rho}$ are not canonical coordinates since the bracket between $p$ and $\psi$ does not vanish. From now on we transform the Greek vector index of $\psi^{\nu}$ into a latin one using the vielbein, $\psi^{a}=e_{\nu}^{a} \psi^{\nu}$. This because we want to think of it as the spin of the particle, which is defined on locally Lorentzian frames. We quickly remark on the statement made at the beginning of the section, saying that $\psi^{\nu}$ represents the spin of the particle. Given the Poisson-Dirac brackets (2.10), these are (modulo constants) the same describing the Clifford algebra of Gamma matrices. Therefore, one readily sees that the operator $\psi^{a} \psi^{b}$ generates rotations on the spin vector via the Poisson-Dirac bracket, and 
that $R_{\mu \nu}$ as defined before generates transformations belonging to the holonomy group of the manifold.

In terms of the covariant momentum $\Pi_{\mu}=p_{\mu}-i / 2 \omega_{\mu a b} \psi^{a} \psi^{b}=\dot{x}_{\mu}$ (this formula becomes evident rewriting the Lagrangian in terms of $\psi^{a}$ ) it is possible to write the Hamiltonian $H$ as

$$
H=\frac{1}{2} g^{\mu \nu} \Pi_{\mu} \Pi_{\nu}
$$

and the Poisson-Dirac bracket of two functions $F, G$ in the covariant way

$$
\{F, G\}=\mathcal{D}_{\mu} F \frac{\partial G}{\partial \Pi_{\mu}}-\frac{\partial F}{\partial \Pi_{\mu}} \mathcal{D}_{\mu} G+R_{\mu \nu} \frac{\partial F}{\partial \Pi_{\mu}} \frac{\partial G}{\partial \Pi_{\nu}}+i(-1)^{\operatorname{deg} F} \frac{\partial F}{\partial \psi^{a}} \frac{\partial F}{\partial \psi_{a}},
$$

where the covariant derivative of a function is

$$
\mathcal{D}_{\mu} F=\partial_{\mu} F+\Gamma_{\mu \lambda}^{\rho} \Pi_{\rho} \frac{\partial F}{\partial \Pi_{\lambda}}+\psi^{a} \omega_{\mu a}{ }^{b} \frac{\partial F}{\partial \psi^{b}} .
$$

From this and (2.12) it is seen that the phase space of the system is defined by a bundle which has $\left(\Pi_{\mu}, \psi_{\nu}\right)$ as coordinates for its fibers.

The spin constraint (2.8) together with the fact that the particle trajectory is null is compatible with the equations of motion since such conditions are equivalent to setting $H$ and $\mathcal{Q}=\Pi_{\mu} \psi^{\mu}$ equal to constants.

\subsection{Supersymmetry and Yano tensors}

The Lagrangian (2.7) is invariant under supersymmetric transformations generated by the Grassmann odd supercharge $\mathcal{Q}$, which amount to

$$
\begin{aligned}
& \delta x^{\mu}=i \epsilon\left\{\mathcal{Q}, x^{\mu}\right\}=-i \epsilon \psi^{\mu}, \\
& \delta \psi^{a}=i \epsilon\left\{\mathcal{Q}, \psi^{a}\right\}=\epsilon \dot{x}^{a}-\delta x^{\mu} \omega_{\mu b}^{a} \psi^{b} .
\end{aligned}
$$

It is also invariant under chiral symmetry, generated by

$$
\gamma_{*}=-\frac{i^{[D / 2]}}{D !} \eta_{a_{1} \ldots a_{D}} \psi^{a_{1}} \ldots \psi^{a_{D}}
$$

where $\eta_{a_{1} \ldots a_{D}}$ is the antisymmetric tensor (not density), and dual supersymmetry, generated by

$$
\mathcal{Q}^{*}=i\left\{\mathcal{Q}, \gamma_{*}\right\}=-\frac{i^{[D / 2]}}{(D-1) !} \eta_{a_{1} \ldots a_{D}} \Pi^{a_{1}} \psi^{a_{2}} \ldots \psi^{a_{D}} .
$$

$H, \mathcal{Q}, \mathcal{Q}^{*}$ and $\gamma_{*}$ provide the only generic symmetries of the theory, i.e. those that exist for every spacetime, see 18, 19].

Additional non generic supersymmetries can be constructed in spacetimes which admit Yano tensors. By definition a Yano tensor of rank $r$ is an antisymmetric tensor

$$
f_{\mu_{1} \ldots \mu_{r}}=f_{\left[\mu_{1} \ldots \mu_{r}\right]}
$$

such that the further condition

$$
\nabla_{\left(\mu_{1}\right.} f_{\left.\mu_{2}\right) \mu_{3} \ldots \mu_{r+1}}=0
$$


holds. This is equivalent to say that $f_{\lambda \mu_{1} \ldots \mu_{r-1}} \dot{x}^{\lambda}$ is parallely propagated along geodesics ${ }^{1}$. Yano has studied these tensors and found conditions the Riemann tensor has to satisfy in order for the spacetime to admit any ([2]). In [6, 7] it has been shown that the only additional non generic supercharges that are also supersymmetric can be constructed from Yano tensors using the formula

$$
Y_{r}=\Pi^{\mu_{1}} f_{\mu_{1} \ldots \mu_{r}} \psi^{\mu_{2}} \ldots \psi^{\mu_{r}}-\frac{i}{r+1} \nabla_{\mu_{1}} f_{\mu_{2} \ldots \mu_{r+1}} \psi^{\mu_{1}} \ldots \psi^{\mu_{r+1}} .
$$

Analogously to Killing vectors (which are Yano tensors of rank 1), Yano tensors satisfy an integrability condition that comes from eqs. 2.18), (2.19):

$$
\nabla_{a} \nabla_{b} f_{\mu_{1} \ldots \mu_{r}}=(-1)^{r+1} \frac{r+1}{2} R_{a\left[b \mu_{1}\right.}^{\lambda} f_{\left.\mu_{2} \ldots \mu_{r}\right] \lambda} .
$$

A typical example of rank 2 Yano tensor is given in flat four dimensional Minkowski space, where the associate conserved quantity has the interpretation of an angular momentum. Let $v^{\mu}$ be a timelike vector associated to the tangent of an observer's worldline, then the Yano tensor is

$$
f_{\mu \nu}=v^{\lambda} \eta_{\lambda \rho \mu \nu} x^{\rho}=\epsilon_{\rho \mu \nu} x^{\rho},
$$

where we have defined $\epsilon_{\rho \mu \nu}=v^{\lambda} \eta_{\lambda \rho \mu \nu}$. Then the conserved quantity $f_{\mu \nu} \Pi^{\mu}=\epsilon_{\rho \mu \nu} x^{\rho} \dot{x}^{\nu}$ has the clear interpretation of an angular momentum as seen by the observer. This is the reason why sometimes quantities associated to rank 2 Yano tensors are referred to as generalized angular momenta.

The supercharges (2.20) generate an exotic extended superalgebra, in the sense that anticommutators of two supercharges do not necessarily close on the Hamiltonian. The case of $\mathcal{N}=q$ supersymmetry generated by rank two Yano tensors $f_{i}^{\mu \nu}, i=1, \ldots, q$ has been studied in [6]. The result is

$$
\left\{\mathcal{Q}_{i}, \mathcal{Q}_{j}\right\}=-2 i Z_{i j},
$$

where the conserved charge $Z_{i j}$ is given by

$$
\begin{aligned}
Z_{i j} & =\frac{1}{2} K_{i j}^{\mu \nu} \Pi^{\mu} \Pi^{\nu}+(\text { spin corrections }), \\
K_{i j}^{\mu \nu} & =\frac{1}{2}\left(f_{i \lambda}^{\mu} f_{j}^{\nu \lambda}+f_{j \lambda}^{\mu} f_{i}^{\nu \lambda}\right) .
\end{aligned}
$$

$K_{i j}^{\mu \nu}$ is a Killing vector, as it is easy to check using (2.18), 2.19), and $Z_{i j}$ the associated conserved quantity for a particle with spin. The vielbein $e_{\mu}^{a}$, once transformed into an object with both indices curved (that is, the metric), though not antisymmetric still defines via (2.20) the conserved charge $\mathcal{Q}$, such that $\{\mathcal{Q}, \mathcal{Q}\}=-2 i H$. When one of these Killing tensors is different from the metric tensor and invertible, then there exists a dual spacetime where $K^{\mu \nu}$ is an (inverse) metric and $g^{\mu \nu}$ a Killing tensor. Then it can be seen that the

\footnotetext{
${ }^{1}$ In the introduction we argued that the absence of quantum anomalies could be due to the fact there exists an underlying structure that does not depend on the metric. As can be seen from (2.19), Yano tensors do actually depend on the metric. However, the Dirac operator depends on the metric as well, and therefore the metric independent structure must result by considering Dirac and Yano operators together.
} 
Yano tensor associated to $K^{\mu \nu}$ becomes a vielbein in the dual space, while the vielbein corresponds to a Yano tensor (see for example [20], [21]). Not always the classical symmetry associated to a general Killing tensor will survive at the quantum level, as we will see in the next section.

\subsection{The quantum system}

In this section we discuss the quantization of the spinning particle. First we show how it implies the Dirac equation. Then in the following we define quantum operators $\hat{Y}_{r}$ which correspond to the classical conserved charges of (2.20), and calculate the (anti)commutator $\left[\not D, \hat{Y}_{r}\right\}$, where $\not D=\Gamma^{\mu} d_{\mu}$ is the Dirac operator. The (anti)commutator is identically zero for every $r$. We comment on the relevance of this result to the theory of the spinning particle: the extra supersymmetries are actually constraints on the Hilbert space of the system that reduce the number of its degrees of freedom.

As already mentioned above, the lagrangian (2.7) can be obtained by a super reparametrization invariant lagrangian after a gauge fixing ([16]) that amounts to a mass shell condition

$$
2 H=g^{\mu \nu} \Pi_{\mu} \Pi_{\nu}=0
$$

and a condition of transversality for the spin

$$
Q=\psi^{\mu} \Pi_{\mu}=0
$$

This describes the massless case. In the quantum theory $H$ and $Q$ will become operators and we can relax the assumption by simply asking that they are constant on the Hilbert space of the system: $Q= \pm m, H=-m^{2}$. There are two ways to quantize the PoissonDirac brackets (2.12). As a specific example we consider the case where the spacetime $M$ is four dimensional, but the same procedure apply in generic dimension. The first type of quantization consists in choosing

$$
\psi_{\mu}=\frac{1}{\sqrt{2}} \Gamma_{\mu},
$$

where $\Gamma_{\mu}$ are the gamma matrices. In this case the algebra of Grassmannian variables is substituted by the Clifford algebra, which is richer. Basic (anti)commutators then are

$$
\begin{aligned}
{\left[\hat{q}_{\mu}, \hat{p}_{\nu}\right] } & =i \hbar g_{\mu \nu}, \\
\left\{\Gamma_{\mu}, \Gamma_{\nu}\right\} & =2 \hbar g_{\mu \nu},
\end{aligned}
$$

where we have made explicit the factor $\hbar$ in order to keep track of it along the following calculations. The covariant momentum operator $\Pi_{\mu}$ is represented by the spinor covariant derivative $D_{\mu}=\partial_{\mu}+\frac{1}{4} \omega_{\mu}^{a b} \Gamma_{a b}$. The constraints amount to $p^{2}=-m^{2}$ the first, while the second one becomes

$$
\Gamma^{\mu} D_{\mu}|\psi>= \pm m| \psi>,
$$

that is, this is the way the Dirac equation in spacetime arises in the theory. The situation is similar to that of the superstring where both spacetime fermions and bosons arise as 
states of the system. As a matter of fact the spinning particle as well has a bosonic sector which is quantized by taking

$$
\psi_{\mu}=\frac{1}{2}\left(a_{\mu}+a_{\mu}^{\dagger}\right)
$$

for two operators $a, a^{\dagger}$ that satisfy

$$
\begin{aligned}
& \left\{a_{\mu}, a_{\nu}^{\dagger}\right\}=\hbar g_{\mu \nu}, \\
& \left\{a_{\mu}, a_{\nu}\right\}=0=\left\{a_{\mu}^{\dagger}, a_{\nu}^{\dagger}\right\} .
\end{aligned}
$$

Then the gauge fixing amounts to select a physical subspace of the full Hilbert space generated by vectors $\mid \psi>$ such that

$$
\begin{aligned}
p^{2} \mid \psi> & =-m^{2}, \\
p_{\mu} a^{\mu} \mid \psi> & = \pm m \mid \psi>,
\end{aligned}
$$

for example, for $m=0$ states are massless and transverse. Supersymmetry on the worldsheet does not necessarily imply supersymmetry in target space. Take $m \neq 0$ for example. If spacetime has $D$ dimensions the bosonic excitations are $p$-forms, $0 \leq p \leq D$, and the total number of degrees of freedom is given by $\Sigma_{n}\left(\begin{array}{c}D-1 \\ n\end{array}\right)=2^{D-1}$. Massive fermions instead have $2^{[D / 2]}$ degrees of freedom, or less if some Weyl or Majorana condition is used. The two numbers coincide only for $D=1,2$. If $m=0$ instead fermionic degrees of freedom are the same while bosonic ones are halved, and they coincide for $D=3,4$. Hence, unless some kind of GSO projection is employed, in all the other cases there is no target space supersymmetry.

Lastly we note that, even if the conservation law $\{\mathcal{Q}, H\}=0$ is satisfied for the quantum system, it is in general not true that the operator associated to the classical charge (2.24) will commute with the Hamiltonian. For example, even for a scalar particle this does not happen. One can construct the operator $K=K^{\mu \nu} D_{\mu} D_{\nu}$, where $D_{\mu}$ is the scalar covariant derivative. The Hamiltonian of the scalar particle is given by $H=1 / 2 g^{\mu \nu} D_{\mu} D_{\nu}$, and calculating the commutator $[K, H]$ one finds a quantum anomaly proportional to

$$
[K, H] \sim K_{[\mu}^{\lambda} R_{\nu] \lambda}
$$

where $R_{\mu \nu}$ is the Ricci tensor. Therefore in general if the spacetime is curved the classical conservation law is violated quantum mechanically. Operators constructed from symmetric tensors are in general a source of anomaly. However, as noted in [5], if $K^{\mu \nu}$ is the square of a rank 2 Yano tensor then the contribution (2.37) is identically zero due to the integrability condition (2.21). This property is actually true for a Yano tensor of arbitrary rank. In the next section we will show that the classical supercharges (2.20) are always conserved even at the quantum mechanical level.

\subsection{Yano operators}

When the spacetime admits some Yano tensors $f$ of rank $r$, one can construct the quantities $Y_{r}$ as in (2.20). They are defined in the symplectic space of the system and classically 
commute both with the supercharge $\mathcal{Q}$, which quantum mechanically corresponds to the Dirac operator (eq.(2.28) ), and its square the Hamiltonian. In quantizing the system to the $Y_{r}$ 's there remain associated operators $\hat{Y}_{r}$ given by

$$
\hat{Y}_{r}=(i)^{\left[\frac{r+1}{2}\right]}\left[\Gamma^{\mu_{1} \ldots \mu_{r-1}} f_{\mu_{1} \ldots \mu_{r-1} \mu_{r}} \hbar D_{\mu_{r}}-\frac{(-1)^{r}}{2(r+1)} \Gamma^{\mu_{1} \ldots \mu_{r+1}} \nabla_{\mu_{1}} f_{\mu_{2} \ldots \mu_{r+1}}\right],
$$

where $D_{\mu}$ is the spinor covariant derivative and $\Gamma^{\mu_{1} \ldots \mu_{n}}$ is the antisymmetrized product of $n$ Gamma matrices with unit weight. For $r$ even these operators contain an odd number of Gamma matrices and are intrinsically 'fermionic', while for $r$ odd they are 'bosonic'. The factor of $i$ is chosen so that in positive definite signature the operators are self-adjoint. In Lorentzian signature there's always an anti self-adjoint part proportional to $\Gamma^{0}$. The following lemma holds.

Lemma. Let $(M, g)$ be a manifold with spin structure and let $f$ be a Yano tensor of rank $r \geq 1$, with associated operator $\hat{Y}_{r}$ as in (2.38). Given the Dirac operator $\not D=\Gamma^{\mu} D_{\mu}$ then

$$
\left[\not D, Y_{r}\right\} \equiv 0
$$

where [ , \} is the commutator for $r$ odd and the anticommutator for $r$ even.

Proof: We delegate the proof to the appendix.

Therefore all the classical extra supersymmetries of the theory of the spinning particle extend to quantum mechanical symmetries. The implication of the lemma is even deeper in that it means that on spacetimes admitting Yano tensors it is always possible to form conserved charges for the Dirac equation. Geometrical properties of spacetime and particle physics have so far always being linked. Consider for example Kaluza Klein compactifications of supergravity. Then the isometries of the compact part of spacetime determine the number of massless gauge bosons, while its holonomy group determines the number of supersymmetries and of massless gravitinos. Now the next question is: what is the geometrical meaning of the conserved quantities associated to Yano tensors, and what is their role in particle physics? Yano tensors can exist on spaces which have no isometries at all. For example six dimensional almost Kähler spaces admit Yano tensors of all ranks from 1 to 5 .

However, the situation is quite different from that of ordinary quantum field theory. While in quantum field theory the supercharges carry spacetime spinor indexes and acting with them on states of the system corresponds to moving in a determined target space supermultiplet, in the spinning particle system the supercharges are target space scalars and do not change the spin of a particle. They are associated to a split of each eigenspace of the Hamiltonian into a sum of two-dimensional eigenspaces on which the supercharge, opportunely rescaled, acts as the group $\mathbb{Z}_{2}$. Then eigenstates of $Q$, which correspond to classical trajectories of the particle with a fixed conserved number, are given by linear combinations of such states.

In the rest of the paper we start analysing a class of spacetimes which admit Yano tensors: spaces of almost special holonomy. Among these, we will construct explicitly Yano tensors on maximally symmetric spaces. On spaces of almost special holonomy one can 
show that it is present a tower of antisymmetric tensors. These will prove to be either Yano, or conformal Yano tensors. These latter objects satisfy a generalized Yano equation, the conformal Yano equation. Yano tensors and conformal Yano tensors are related by Hodge duality: as we show in the next section, the conformal Yano equation is duality invariant.

\section{Conformal Yano equation and Hodge duality}

Generalizing the construction of eq.(2.25), given two rank $r$ Yano tensors $f_{\mu_{1} \ldots \mu_{r}}, g_{\mu_{1} \ldots \mu_{r}}$, it is possible to associate to them the symmetric rank 2 tensor

$$
K_{\mu \nu}^{(f, g)}:=\left(f_{\mu \lambda_{2} \ldots \lambda_{r}} g_{\nu}^{\lambda_{2} \ldots \lambda_{r}}+g_{\mu \lambda_{2} \ldots \lambda_{r}} f_{\nu}^{\lambda_{2} \ldots \lambda_{r}}\right) .
$$

Eq.(2.19) then ensures that $K^{(f, g)}$ is a Killing tensor, i.e. it satisfies

$$
\nabla_{(\lambda} K_{\mu \nu)}^{(f, g)}=0 .
$$

It is possible to use the same reasoning in order to construct conformal Killing tensors. A conformal Killing tensor $K_{\mu \nu}$ is by definition a symmetric tensor satisfying the equation

$$
\nabla_{(\lambda} K_{\mu \nu)}=g_{(\lambda \mu} a_{\nu)} .
$$

Note that $K_{\mu \nu}+\alpha g_{\mu \nu}$ satisfies the same equation for any given constant $\alpha$ and so we can always take $K$ to be traceless. In this case then $a_{\mu}=2 /(D+2) \nabla^{\lambda} K_{\lambda \mu}$. As Killing tensors generate conserved charges of the system, i.e. functions which commute with the Hamiltonian, conformal Killing tensors generate functions whose commutator with $H$ is proportional to $H$ itself. The conformal Yano equation has been discussed by Tachibana [22]. In the case of rank 2 it is given by

$$
\nabla_{(\lambda} f_{\mu) \nu}=g_{\lambda \mu} \phi_{\nu}-g_{\nu(\lambda} \phi_{\mu)}
$$

where $\phi_{\mu}=1 / 3 \nabla_{\lambda} f_{\mu}^{\lambda}$. Four dimensional spacetimes admitting a conformal Yano tensor of rank 2 must be of Petrov type D, N or O (see for example [23]). Moreover, eq.(3.4) is invariant in form under Hodge duality (24]).

Now we generalize the construction to conformal Yano tensors of higher rank in arbitrary dimension. We can start by a definition similar to that of eq.(3.4):

$$
\nabla_{\left(\mu_{1}\right.} f_{\left.\mu_{2}\right) \mu_{3} \ldots \mu_{r+1}}=g_{\mu_{1} \mu_{2}} \Phi_{\mu_{3} \ldots \mu_{r+1}}+A g_{\left[\mu _ { 3 } \left(\mu_{1}\right.\right.} \Phi_{\left.\left.\mu_{2}\right) \mu_{4} \ldots \mu_{r+1}\right]},
$$

where $A$ is a constant and $\Phi=1 /(D+A) \nabla f$. This definition ensures that the tensor $K^{(f, f)}$ as in eq.(3.1) satisfies the Killing conformal equation with $a_{\nu}=2 f_{\nu \lambda_{1} \ldots \lambda_{r-1}} \Phi^{\lambda_{1} \ldots \lambda_{r-1}}$ independently of the constant $A$. However, in general if $f, g$ are two rank $r$ antisymmetric tensors and both satisfy eq.(3.5), then $K^{(f, g)}$ is not conformal Killing unless for both $f$ and $g$ the constant $A$ is equal to zero.

In the appendix we show that the constant $A$ is fixed by consistency with Hodge duality to be equal to $(1-r)$, and that, moreover, in this case the dual of $f$ satisfies the same equation, that is, the conformal Yano equation

$$
\nabla_{\left(\mu_{1}\right.} f_{\left.\mu_{2}\right) \mu_{3} \ldots \mu_{r+1}}=g_{\mu_{1} \mu_{2}} \Phi_{\mu_{3} \ldots \mu_{r+1}}-(r-1) g_{\left[\mu _ { 3 } \left(\mu_{1}\right.\right.} \Phi_{\left.\left.\mu_{2}\right) \mu_{4} \ldots \mu_{r+1}\right]}
$$


is preserved by duality. Yano tensors themselves are a subset of all conformal Yano tensors, those for which $\Phi=0$ (co-closed). Whenever the spacetime admits a Yano tensor, then its dual is a conformal Yano tensor.

\section{Spaces of almost special holonomy}

Let $M$ be a spacetime of dimension $D$ which admits a spin structure and $S$ a spin bundle over $M$. If a Killing spinor exists on $S$ then its holonomy is reduced with respect to a connection that extends the usual Levi-Civita spinorial connection: the space is said to be of almost special holonomy. In this situation it is possible to construct from it a tower of tensors on $M$, which happen to split into genuinely Yano tensors and conformal Yano tensors. Hodge duality mixes them along the lines of section (3). The local form of Yano and conformal Yano tensors in pseudo-Riemannian spaces of constant curvature has been studied in 25.

We start from the definition of a Killing spinor, which is covariantly constant with respect to the extended derivative

$$
\mathcal{D}_{\mu}^{ \pm} \eta^{ \pm}:=\left(D_{\mu} \mp i \lambda \Gamma_{\mu}\right) \eta^{ \pm}=0
$$

where $D_{\mu}$ is the spinorial covariant derivative. If $\lambda=0$ then this is the equation for a covariantly constant spinor, and all the tensors one can construct from it are covariantly constant.

It is worthwhile spending a few lines to comment on the integrability conditions of (4.1) for two reasons. One is that in this section we are going to construct special tensors on spacetimes admitting Killing spinors and therefore we are interested in knowing to which cases the construction applies. The second one is that in the next section we are going to solve the Killing equation for maximally symmetric spacetimes, which result to be those that admit the maximum number of solutions. Let's start with the case $\lambda=0$, that is, covariantly constant spinors. Then one has

$$
0=\left[D_{\mu}, D_{\nu}\right] \eta=\frac{1}{4} R_{\mu \nu \rho \sigma} \Gamma^{\rho \sigma} \eta
$$

Multiplying on the left by $\Gamma^{\nu}$ one gets the condition

$$
R_{\mu \nu} \Gamma^{\nu} \eta=0
$$

whose square gives

$$
\begin{aligned}
R_{\mu}^{\lambda} R_{\nu \lambda} & =0, \\
R & =0 .
\end{aligned}
$$

(4.3), (4.4), (4.5) give conditions on the Ricci tensor and the curvature scalar. There is also a condition on the Weyl tensor that comes from eq.(4.2), amounting to

$$
\frac{1}{4} C_{\mu \nu \rho \sigma} \Gamma^{\rho \sigma} \eta=0
$$


In Euclidean signature (4.4) implies that spacetime is Ricci flat and the holonomy of the spin connection $D_{\mu}$ is reduced. Such spaces are well known. In particular, if $M$ is simplyconnected and its metric is irreducible and non symmetric, there applies a classification due to Berger: depending on the number of covariantly constant spinors and the dimension of spacetime one can get the holonomy group to be $S U(D / 2)$ in even dimension (Calabi-Yau), $\operatorname{Sp}(D / 4)$ (hyperKähler) for $D=4 m$ and the exceptional cases $G_{2}$ for $D=7, \operatorname{Spin}(7)$ for $D=8$ (see [26] for example).

If the signature is Lorentzian instead (4.4) does not imply that the Ricci tensor is zero. A counterexample is given by the $p p$-wave spacetimes, whose Ricci tensor is null. $p p$-wave spacetimes have $R=0$ and eqs.(4.3), (4.6) become a null projection, so that the number of covariantly constant spinors is at most half the maximum number admitted. When the $p p$-wave spacetime is of Kowalski-Glikman kind then it is a symmetric space ([27).

Now consider $\lambda \neq 0$. Conditions analogous to (4.3), (4.4), (4.6) are

$$
\begin{aligned}
R_{\mu \nu} \Gamma^{\nu} \eta & =\left(2(D-2) \lambda^{2}+\frac{R}{2(D-1)}\right) \Gamma_{\mu} \eta \\
R_{\mu}^{\lambda} R_{\nu \lambda} & =\left(2(D-2) \lambda^{2}+\frac{R}{2(D-1)}\right) R_{\mu \nu}, \\
\frac{1}{4} C_{\mu \nu \rho \sigma} \Gamma^{\rho \sigma} \eta & =0 .
\end{aligned}
$$

These reduce to eqs.4.3), (4.4), (4.5) by taking $\lambda=0, R=0$. In Euclidean signature (4.8) implies that the space is Einstein with constant scalar curvature $R=4 D(D-1) \lambda^{2}$. All these spaces are locally symmetric and can be fully described.

$\lambda$ real implies that $M$ is compact with positive curvature, and can be classified by the holonomy of its cone, where the cone over $M$ is defined as the space with metric $d s^{2}=d r^{2}+r^{2} d s_{M}^{2}$. It is not important whether the cone has any singularity at $r=0$ or not, since one is simply interested in rewriting the Killing equation on $M$ into a simpler equation on a different space. Therefore, all it is necessary to do is to classify the possible cones admitting covariantly constant spinors. The standard approach is to reduce this procedure to Berger's classification: if one assumes $M$ is simply connected (but this will not be true in general), then a result of Gallot ([29]) says that the cone over $M$ is either flat, or irreducible. A flat cone, outside the origin $r=0$, can be obtained not only if $M$ is a $D$-dimensional round sphere, but for all spaces that are locally a sphere, that is maximally symmetric spaces. The sphere is the only space such that its cone has no singularities at the origin, but maximal symmetry is a sufficient condition for flatness outside $r=0$. If the cone is irreducible instead, one can have 3-Sasaki manifolds for $D=4 m-1$ and hyperKähler cone, Sasaki-Einstein for $D=4 m \pm 1$ and cone Calabi-Yau, almost Kähler for $D=6$ and cone $G_{2}$, almost $G_{2}$ for $D=7$ and cone $\operatorname{Spin}(7)$ (see for example [30], [31]).

For $\lambda$ imaginary $M$ is non compact with negative curvature. Then it can be shown that either $M$ is the hyperbolic plane $H^{D}$ or it is given by a warped product $M=N \times \mathbb{R}$ with metric $d s^{2}=e^{\mu t} d s_{N}^{2}+d t^{2}$, where $N$ is a complete, connected spin manifold which admits non trivial parallel spinors and $\mu \in \mathbb{R} \backslash\{0\}$. Conversely, every such $N$ gives, when warped, a manifold $M$ with imaginary Killing spinors ([31]). 
In Lorentzian signature instead the spacetime is not necessarily Einstein and there is much more freedom. If we restrict our attention to the subclass of Einstein spaces then it is possible again to relate $\lambda$ and $R$. For $\lambda$ real the curvature is positive, even if no compactedness is required, and for example the analogue of the Euclidean sphere is given by de Sitter space. For $\lambda$ imaginary the curvature is negative and the analogue of the hyperbolic plane is Anti-de Sitter space. The case $\lambda$ imaginary has been recently studied in 32.

Because of the integrabililty conditions we just discussed, in general only a subset of all the possible spinors on $M$ will satisfy (4.1). For example, if there exists a solution of the kind $\eta^{+}$, there is no reason in general to have also a solution of the kind $\eta^{-}$, and vice versa (for maximally symmetric spaces both exist). The subset of Killing spinors will be selected by using an appropriate projection matrix. This implies that, of all the tensor we are going to construct below, only part of them will be non zero. However, to keep the maximum generality, we will discuss all the possible cases.

We are now in the position to construct special tensors on spaces of almost special holonomy. If the dimension $D=2 m$ is even then one can construct a chirality matrix

$$
\begin{aligned}
\Gamma_{*} & =(-i)^{m+t} \Gamma_{1} \ldots \Gamma_{D}, \\
\Gamma_{*} \Gamma_{*} & =1 .
\end{aligned}
$$

$t$ is zero in Euclidean signature and one in Lorentzian, which we define using an almost plus convention. The chirality matrix satisfies

$$
\Gamma_{*} \mathcal{D}^{ \pm}=\mathcal{D}^{\mp} \Gamma_{*}
$$

and therefore the two kinds of Killing spinors can be related by

$$
\eta^{-}=\Gamma_{*} \eta^{+},
$$

and they are not eigenspinors of chirality.

Regardless of the dimension and the signature one can define the following $n$-forms

$$
\begin{aligned}
& A_{\left[\mu_{1} \ldots \mu_{n}\right]}^{(n)}:=\bar{\eta}^{+} \Gamma_{\mu_{1} \ldots \mu_{n}} \eta^{-}, \\
& B_{\left[\mu_{1} \ldots \mu_{n}\right]}^{(n)}:=\bar{\eta}^{+} \Gamma_{\mu_{1} \ldots \mu_{n}} \eta^{+} .
\end{aligned}
$$

We want to show that for each $n=1, \ldots, D-1$ either $A^{(n)}$ or $B^{(n)}$ is a Yano tensor and the other one is a conformal Yano. Moreover, if $A^{(n)}$ is Yano then $A^{(n+1)}$ is conformal Yano, $A^{(n+2)}$ Yano again and so on, and the same for $B^{(n)}$.

In the case of covariantly constant spinors these forms are known to generate calibrations in supergravity theories without $p$-form flux. In the presence of flux the same forms can be used to define calibrations, but the spinors $\eta$ have to satisfy a generalized Killing equation with flux, which we do not contemplate here. It should be interesting to investigate further the connection between Yano tensors and supergravity calibrations.

Note that condition $D_{\mu} \eta^{+}=i \lambda \Gamma_{\mu} \eta^{+}$implies

$$
D_{\mu} \bar{\eta}^{+}=(-1)^{t+1} i \lambda^{*} \bar{\eta}^{+} \Gamma_{\mu},
$$


so that in the following discussion the tensors $A$ and $B$ will reverse their role depending whether $t=0,1$ and whether $\lambda$ is real or imaginary. Moreover, in case $\eta^{ \pm}$spinors are more than one we will distinguish them by an index $\alpha$, and 4.16) implies that it is possible to normalize them. In Euclidean signature, and for $\lambda$ real (the $D$-dimensional sphere for example), one can take

$$
\bar{\eta}^{\alpha+} \eta_{\beta}^{+}=M_{\beta}^{\alpha},
$$

where $M_{\beta}^{\alpha}$ is a suitable constant matrix. For $\lambda$ imaginary (hyperbolic plane for example) one has to take the opposite combination $\bar{\eta}^{\alpha+} \eta_{\beta}^{-}=M_{\beta}^{\alpha}$, while passing from Euclidean to Lorentzian signature these two cases interchange.

For concreteness from now until the end of this section we now work in Euclidean signature and with $\lambda$ real. To the extent of showing properties of $A$ and $B$ tensors we use the following identities

$$
\begin{aligned}
& \Gamma_{\lambda} \Gamma_{\mu_{1} \ldots \mu_{n}}=\Gamma_{\lambda \mu_{1} \ldots \mu_{n}}+n g_{\lambda\left[\mu_{1}\right.} \Gamma_{\left.\mu_{2} \ldots \mu_{n}\right]}, \\
& \Gamma_{\mu_{1} \ldots \mu_{n}} \Gamma_{\lambda}=\Gamma_{\mu_{1} \ldots \mu_{n} \lambda}+(-1)^{n+1} n g_{\lambda\left[\mu_{1}\right.} \Gamma_{\left.\mu_{2} \ldots \mu_{n}\right]},
\end{aligned}
$$

together with (4.16), to calculate covariant derivatives of $A$ and $B$. Suppose $n=2 k+1$ is odd. Then one has

$$
\nabla_{\lambda} A_{\mu_{1} \ldots \mu_{n}}^{(n)}=-i \lambda \bar{\eta}^{+}\left(\Gamma_{\lambda} \Gamma_{\mu_{1} \ldots \mu_{n}}+\Gamma_{\mu_{1} \ldots \mu_{n}} \Gamma_{\lambda}\right) \eta^{-}=-2 i n \lambda \bar{\eta}^{+} g_{\lambda\left[\mu_{1}\right.} \Gamma_{\left.\mu_{2} \ldots \mu_{n}\right]} \eta^{-},
$$

from which it is readily seen that

$$
\nabla_{(\lambda} A_{\left.\mu_{1}\right) \mu_{2} \ldots \mu_{n}}^{(n)}=-2 i n \lambda\left[g_{\lambda \mu_{1}} \bar{\eta}^{+} \Gamma_{\mu_{2} \ldots \mu_{n}} \eta^{-}-(n-1) g_{\left[\mu_{2}(\lambda\right.} \bar{\eta}^{+} \Gamma_{\left.\left.\mu_{1}\right) \mu_{3} \ldots \mu_{n}\right]} \eta^{-}\right]
$$

that is $A^{(2 k+1)}$ satisfies the conformal Yano equation and is not a strictly Yano tensor. However, from $(4.20)$ it is immediate to check that $A^{(2 k+1)}$ is closed and therefore its Hodge dual is strictly Yano. The same calculation for $B^{(2 k+1)}$ yields

$$
\begin{aligned}
\nabla_{\lambda} B_{\mu_{1} \ldots \mu_{n}}^{(n)} & =i \lambda \bar{\eta}^{+}\left(-\Gamma_{\lambda} \Gamma_{\mu_{1} \ldots \mu_{n}}+\Gamma_{\mu_{1} \ldots \mu_{n}} \Gamma_{\lambda}\right) \eta^{+}=-2 i n \lambda \bar{\eta}^{+} \Gamma_{\lambda \mu_{1} \ldots \mu_{n}} \eta^{+} \\
& =-2 i \lambda B_{\lambda \mu_{1} \ldots \mu_{n}}^{n+1}
\end{aligned}
$$

Therefore $B^{(2 k+1)}$ is strictly Yano but, since it is not closed in general, its Hodge dual will be conformal Yano. For $n=2 k$ even instead the identities eq.(4.18),(4.19) imply that $A$ and $B$ exchange their roles: therefore $A^{(2 k)}$ becomes Yano and $B^{(2 k)}$ conformal Yano. Therefore one gets two towers of tensors of increasing rank which are alternatively Yano and conformal Yano. Yano tensors which are covariantly constant are more special than the others since they generate symmetries that extend to String Theory. It is not easy to give a general rule for the tensors of $\mathrm{A}$ and $\mathrm{B}$ kind to be covariantly constant. Clearly it is true when the Killing spinor is itself covariantly constant, or it can happen when the Killing equation gets modified by a flux term, like for pp-wave spacetimes. In absence of further information however a general spinor will not necessarily give covariantly constant Yano tensors. One should take into account other projections on the spinor, if they occur.

It is conventional to call a spinor satisfying (4.1) Killing, since it generates Killing vectors. However, Killing vectors happen to coincide with Yano tensors of rank 1 and, 
given that the whole tower of tensors constructed from such a spinor is made of (conformal) Yano tensors, a more appropriate name would rather be that of Yano spinor.

Notice that in this setting we are dealing with constructing conformal Yano tensors as covariant derivatives of Yano tensors. This is not allowed in the general case because of the integrability condition (2.21), but is possible on spacetimes with Killing spinors.

One further remark is that $A$ and $B$ tensors have a complementary behaviour which is required by consistency with Hodge duality when the dimension is even. When $D=2 m$ the Hodge duality operation sends tensors of odd rank into tensors of odd rank, and likewise for even rank, but at the same time it exchanges tensors of kind $A$ and $B$, since it holds

$$
\Gamma_{\mu_{1} \ldots \mu_{n}}=(i)^{m+t} \Gamma_{*} \frac{1}{(D-n) !} \eta_{\mu_{1} \ldots \mu_{n} \nu_{n+1} \ldots \nu_{D}} \Gamma^{\nu_{D} \ldots \nu_{n+1}}
$$

Therefore type $A$ tensors are sent into type $B$ tensors by duality, but we know that duality changes Yano tensors into closed conformal Yano tensors and vice versa. Since covariant derivatives of $A$ and $B$ depend on the algebra of Gamma matrices, and this does not depend on the dimension being even or odd, then one recovers the same complementary structure in odd dimension.

\section{Maximally symmetric spaces}

Maximally symmetric spaces are the simplest case to consider since the absence of extra structures makes the computation of Killing spinors and (conformal) Yano tensors easy. Such spacetimes are exactly those that support (when they are spin manifolds) the maximum number of solutions of the Killing spinor equation. Using group theoretical techniques and Gamma matrix manipulations we give a sufficient condition for a symmetric space to be maximally symmetric, in terms of Killing forms and structure coefficients.

In the following section we give a brief account of some properties of symmetric spaces in general and then show how this can be used to solve the Killing spinor equation when the space is maximally symmetric(see [27] for the case $\lambda \neq 0$, where it is applied to the study of supersymmetry algebras in $A d S^{p} \times S^{q}$ spacetimes). From Killing spinors then we construct conformal Yano tensors, and show they are spanned by products of Killing vectors. This is the maximum possible freedom allowed by the underlying structure.

\subsection{Geometry of symmetric spaces}

Let $M$ be a (connected) symmetric spacetime of dimension $D$. Let a Lie group $G$ be its isometry group, then $\operatorname{dim}(G)=D+h, h \geq 0$. There is a natural action of $G$ on $M$ and the isotropy group $H, \operatorname{dim}(H)=h$, is a Lie group as well. $M$ can be described as the homogeneous space $G / H$. The Lie algebra of $G$ can be written as $\mathbf{g}=\left\{T_{I}\right\}=\left\{M_{i}, P_{a}\right\}$, $I=1, \ldots, D+h, i=1, \ldots h, a=1, \ldots, D$, where the $M_{i}$ are generators of $\mathbf{h}$, the Lie algebra of $H$, and the $P_{a}$ span a complementary algebra $\mathbf{m}$. $\mathbf{h}$ and $\mathbf{m}$ are eigenspaces of a Lie algebra involution with eigenvalues +1 and -1 respectively. Then one can show that 
they have following commutation rules

$$
\begin{aligned}
& {\left[M_{i}, M_{j}\right]=f_{i j}^{k} M_{k},} \\
& {\left[M_{i}, P_{a}\right]=f_{i a}^{b} P_{b},} \\
& {\left[P_{a}, P_{b}\right]=f_{a b}^{i} M_{i} .}
\end{aligned}
$$

The first condition says that $\mathbf{h}$ is a subalgebra of $\mathbf{g}$. The second one that $\mathbf{m}$ is a representation of $\mathbf{h}$. The third one that commutators in $\mathbf{m}$ cannot close in $\mathbf{m}$ itself. The spaces $\mathbf{h}$ and $\mathbf{m}$ are orthogonal with respect to the Killing metric.

A generic element in $G / H$ can be obtained by exponentiating the generators $P_{a}$ :

$$
j(x)=e^{x^{1} P_{1}} \ldots e^{x^{D} P_{D}},
$$

and if a matrix representation for the algebra $\mathbf{g}$ is used,

$$
\begin{aligned}
& M_{i} \rightarrow R\left(M_{i}\right), \\
& P_{a} \rightarrow R\left(P_{a}\right),
\end{aligned}
$$

then a representative $R(j(x))$ is obtained by exponentiating the representatives of the $P_{a}$. Under a constant $g \in G$ transformation the element $g j(x)$ is no longer a coset representative of the form (5.2), unless one applies a compensating multiplication by $h \in H$ on the right, where $h$ is a function of both $g$ and the coordinates $x$ :

$$
j\left(x^{\prime}\right)=g j(x) h^{-1}
$$

An important part of the construction is the adjoint action of $\mathbf{g}$ on any representation

$$
A d j_{T_{I}}\left(R\left(T_{J}\right)\right)=R\left(T_{K}\right) R_{A d j}\left(T_{I}\right)_{J}^{K}:=\left[R\left(T_{I}\right), R\left(T_{J}\right)\right]
$$

which when exponentiated

$$
R_{A d j}(g(y))^{K}=e^{y^{I} R_{A d j}\left(T_{I}\right)^{K}{ }_{J}}
$$

(where $y^{I}$ are coordinates for $G$ ), gives the adjoint action of $G$ on its Lie algebra

$$
R(g) R\left(T_{I}\right) R\left(g^{-1}\right)=R\left(T_{J}\right) R_{A d j}(g)^{J}{ }_{I}
$$

If it possible to define duals $T^{I}$ of the generators by some action $T^{I}\left(T_{J}\right)=\delta_{J}^{I}$, for example when the bi-invariant Killing metric

$$
K_{I J}=\operatorname{Tr}\left[R\left(T_{I}\right) R\left(T_{J}\right)\right]
$$

is non degenerate, then these will transform under the adjoint action of $G$ as

$$
R(g) R\left(T^{I}\right) R\left(g^{-1}\right)=R_{A d j}\left(g^{-1}\right)_{J}^{I} T^{J} .
$$

The last notion we need to introduce is that of a global frame for $M$ and its associated spin connection. The Maurer-Cartan 1-form $\Theta=g^{-1} d g$ is left invariant and takes values 
in g. It provides a global frame for $G$. One can decompose it according to the $G / H$ structure as:

$$
g^{-1} d g=e^{a} P_{a}+\theta^{i} M_{i}
$$

$\left\{e^{a}\right\}$ provides a global frame for $M$ and projecting the Maurer-Cartan equation $d \Theta+\Theta \wedge \Theta$ on the $a$ components one finds

$$
d e^{a}+\theta^{i} \wedge e^{b} f_{i b}^{a}=0
$$

which is the equation for a torsionless connection given by

$$
w^{a}{ }_{b}=\theta^{i} f_{i b}^{a} .
$$

This is in agreement with the fact that under a transformation eq.(5.4) $\theta$ transforms like a connection gauging local $H$ transformations:

$$
\theta^{i}\left(x^{\prime}\right)=\left(h \theta(x) h^{-1}\right)^{i}+\left(h^{-1} d h\right)^{i} .
$$

Given a constant bilinear form $N_{(a b)}$ which is non degenerate one can construct the metric

$$
d s^{2}=N_{a b} e^{a} \otimes e^{b} .
$$

If it satisfies

$$
f_{i(a}^{c} N_{b) c}=0
$$

then it is left invariant under the action of the full group $G$ (this is true for the projection of the Killing metric $K_{I J}$ on $a, b$ indexes when it is non degenerate) and it admits at least $G$ as isometry group. Its associated Killing vectors can be seen to be given by

$$
k_{(I)}^{a}(x)=R_{A d j}\left(j^{-1}(x)\right)^{a} .
$$

\subsection{Yano tensors on maximally symmetric spaces}

In this section we use the procedure of [27 to construct Killing spinors on maximally symmetric spaces. From these we derive Yano tensors.

Start from the Killing equation

$$
0=(\nabla-i \lambda \Gamma) \eta^{+}=\left(d+\frac{1}{4} \omega_{a b} \Gamma^{a b}-i \lambda \Gamma\right) \eta^{+} .
$$

An explicit check shows that

$$
R_{s}\left(M_{i}\right):=\frac{1}{4} f_{i a}^{b} \Gamma_{b}^{a}
$$

provides a spinorial representation of $\mathbf{h}$ so that, using (5.12), one can rewrite the Killing equation as

$$
0=\left(d+\theta^{i} R_{s}\left(M_{i}\right)-i \lambda e^{a} \Gamma_{a}\right) \eta^{+}
$$

Now suppose that the matrix $-i \lambda \Gamma_{a}$ provides a representation of the generators $P_{a}$ :

$$
R_{s}\left(P_{a}\right):=-i \lambda \Gamma_{a}
$$


then the Killing equation can be put in the suggestive form

$$
\begin{aligned}
0 & =\left(d+\theta^{i} R_{s}\left(M_{i}\right)+e^{a} R_{s}\left(P_{a}\right)\right) \eta^{+}=\left(d+R_{s}\left(j^{-1}\right) d R_{s}(j)\right) \eta^{+} \\
& =R_{s}\left(j^{-1}\right) d\left(R_{s}(j) \eta^{+}\right) .
\end{aligned}
$$

This is solved by

$$
\begin{aligned}
\eta^{+\alpha} & =R_{s}\left(j^{-1}\right)^{\alpha}{ }_{\beta} \epsilon^{\beta}, \\
R_{s}(j(x)) & =e^{x^{1} R_{s}\left(P_{1}\right)} \ldots e^{x^{D} R_{s}\left(P_{D}\right)},
\end{aligned}
$$

where $\epsilon$ is a constant spinor.

As can be seen, the number of Killing spinors admitted is maximal and a basis for them is given by

$$
\eta_{(\alpha)}^{+\beta}=R_{s}\left(j^{-1}\right)_{\alpha}^{\beta}
$$

An immediate consequence is that, since the Gamma matrices are invariant tensors of $\operatorname{Spin}(D)$ (or $\operatorname{Spin}(1, D-1)$, depending on the signature), then the basis transforms covariantly under $\operatorname{Spin}(D)(\operatorname{Spin}(1, D-1))$ rotations:

$$
\eta_{(\gamma)}^{+\beta}\left(x^{\mu}\right) U_{\alpha}^{\gamma}=U_{\gamma}^{\beta} \eta_{(\alpha)}^{+\gamma}\left(\Lambda_{\nu}^{(-1) \mu} x^{\nu}\right)
$$

where $U$ is the operator associated to the $S O(D)(S O(1, D-1))$ rotation $\Lambda_{\nu}^{\mu}$. Spacetimes like for example Sasaki-Einstein, almost Kähler, almost $G_{2}$ mentioned in the previous section admit less than the maximum number of Killing spinors and therefore are not described by this approach. The reason is that one of the assumptions we made is that $-i \lambda \Gamma_{a}$ is a representative of the $P_{a}$ generators but, as we will see later, this does not happen in general.

Now we are in the position to discuss the structure of special tensors on these spaces. We restrict our attention to the Euclidean signature case and $\lambda$ real, which encodes all the interesting structure. The only differences one encounters when changing to Lorentzian signature and $\backslash$ or $\lambda$ imaginary are those related to the fact that the hermitian conjugate of the matrix $R\left(P_{a}\right)$ will flip sign if $\lambda$ becomes imaginary and will be sandwiched between two $\Gamma^{0}$ matrices in Lorentzian signature. This is paired to the fact the tensors of kind $A$ and $B$ of the previous section interchange their roles.

First of all let us make contact with (4.17) that says it is possible to normalize the spinors. $(+)$-type solutions of the Killing equation are given by (5.24). Solutions of (-)-type are obtained by changing sign to $\lambda$ or, equivalently, by sending $R_{s}\left(P_{a}\right) \rightarrow-R_{s}\left(P_{a}\right):=R_{-s}\left(P_{a}\right)$. This defines an equivalent representation of the $P_{a}$ generators which is on the same footing as the first one. If $\lambda$ is real then $R_{s}\left(j^{-1}\right)^{\dagger}=R_{s}(j)$, from the definition (5.20). Therefore

$$
\bar{\eta}^{\alpha+} \eta_{\beta}^{+}=\left[R_{s}(j) R_{s}\left(j^{-1}\right)\right]_{\beta}^{\alpha}=\delta_{\beta}^{\alpha} .
$$

If instead $\lambda$ is imaginary then this is no longer true and it holds instead $R_{-s}\left(j^{-1}\right)^{\dagger}=R_{s}(j)$, from which the need to put together $(+)$ and $(-)$ spinors in order to form constants. The same holds in Lorentzian signature with minor changes. 
Now consider the special tensors $A$ and $B$. Write

$$
\begin{aligned}
B_{(\beta)}^{(n)(\alpha)} & :=B_{(\beta)\left[\mu_{1} \ldots \mu_{n}\right]}^{(n)(\alpha)} d x^{\mu_{1}} \ldots d x^{\mu_{n}}=\bar{\eta}^{\alpha+} \Gamma^{a_{1} \ldots a_{n}} \eta_{\beta}^{+} e_{a_{1}} \ldots e_{a_{n}} \\
& =(-i \lambda D)^{n} R_{s}(j) R_{s}\left(P^{\left[a_{1}\right.}\right) \ldots R_{s}\left(P^{\left.a_{n}\right]}\right) R_{s}\left(j^{-1}\right) e_{a_{1}} \ldots e_{a_{n}}
\end{aligned}
$$

where we defined representatives of the dual generators $R_{s}\left(P^{a}\right):=\frac{i}{\lambda D} \Gamma^{a}$ according to

$$
\operatorname{Tr}\left[R_{s}\left(P_{a}\right) R_{s}\left(P^{b}\right)\right]=\delta_{a}^{b}
$$

We can immediately recognize the adjoint action of $G$ on the dual of $\mathbf{m}$, given by eq.(5.9). This allows to rewrite $B$ as

$$
(-i \lambda D)^{n} R_{A d j}\left(j^{-1}\right)_{J_{1}}^{\left[a_{1}\right.} R_{s}\left(T^{J_{1}}\right)_{\lambda_{1}}^{\alpha} \ldots R_{A d j}\left(j^{-1}\right)_{J_{n}}^{\left.a_{n}\right]} R_{s}\left(T^{J_{n}}\right)_{\beta}^{\lambda_{2 n-2}} e_{a_{1}} \ldots e_{a_{n}}
$$

Now we just need to recall that Killing forms can be expressed as in eq.(5.16), and we get the final form

$$
B_{(\beta)}^{(n)(\alpha)}=(-i \lambda D)^{n}\left[M^{\left[J_{1} \ldots J_{n}\right]}\right]_{\beta}^{\alpha} k_{\left(J_{1}\right)} \ldots k_{\left(J_{n}\right)},
$$

where $\left[M^{\left[J_{1} \ldots J_{n}\right]}\right]_{\beta}^{\alpha}$ is the constant matrix

$$
\left[M^{\left[J_{1} \ldots J_{n}\right]}\right]_{\beta}^{\alpha}:=R_{s}\left(T^{\left[J_{1}\right.}\right)_{\lambda_{1}}^{\alpha} \ldots R_{s}\left(T^{\left.J_{n}\right]}\right)_{\beta}^{\lambda_{2 n-2}} .
$$

Therefore we learn that $B$ tensors on these spaces have an extremely simple form which is dictated by the large amount of symmetry: they are spanned by products of Killing forms with constant coefficients. These tensors as well as their spinorial building blocks transform in a representation of $\operatorname{Spin}(D)$, as it can be easily seen by (5.25).

For what concerns tensors of kind $A$ almost nothing changes: one has just to notice that $\bar{\eta}^{\alpha-}=R_{-s}\left(j^{-1}\right)^{\dagger} \neq R_{s}(j)$, so that one gets the same expression as above but multiplied by a prefactor

$$
\begin{aligned}
A_{(\beta)}^{(n)(\alpha)} & =(-i \lambda D)^{n}\left[R_{-s}\left(j^{-1}\right)^{\dagger} R_{s}\left(j^{-1}\right)\right]_{\gamma}^{\alpha}\left[M^{\left[J_{1} \ldots J_{n}\right]}\right]_{\beta}^{\gamma} k_{\left(J_{1}\right)} \ldots k_{\left(J_{n}\right)} \\
& =\left[R_{-s}\left(j^{-1}\right)^{\dagger} R_{s}\left(j^{-1}\right)\right]_{\gamma}^{\alpha} B_{(\beta)}^{(n)(\gamma)},
\end{aligned}
$$

that is, $A$ and $B$ tensors are related by an invertible, although position dependent, transformation, and are equivalent.

All the discussion easily carries over the Lorentzian case, where simply the roles of $A$ and $B$ tensors are exchanged.

We conclude this session with a discussion of the assumption (5.20). If the Gamma matrices define a representation of the generators $P_{a}$ then they must satisfy

$$
\left[R_{s}\left(P_{a}\right), R_{s}\left(P_{b}\right)\right]=f_{a b}^{i} R_{s}\left(M_{i}\right)=\frac{1}{4} f_{a b}^{i} f_{i c}^{d} \Gamma_{d}^{c} .
$$

At the same time, the definition (5.20) implies

$$
\left[R_{s}\left(P_{a}\right), R_{s}\left(P_{b}\right)\right]=-2 \lambda^{2} \Gamma_{a b},
$$


and these two conditions are consistent if

$$
f_{a b}^{i} f_{i c}^{d}=-8 \lambda^{2} \delta_{[a}^{d} N_{b] c}
$$

In case the Killing form is invertible it is possible to massage further this equation: multiply on the right by $f_{j d}^{c}$ and use

$$
K_{i j}=f_{i c}^{d} f_{j d}^{c}+f_{i l}^{m} f_{j m}^{l}=f_{i c}^{d} f_{j d}^{c}+K_{i j}^{H},
$$

where $K^{H}$ is the Killing form of the group $H$. A little algebra shows that (5.35) amounts to

$$
\left\{\begin{array}{l}
K_{i j}^{H}=\alpha K_{i j}, \\
K_{a b}=\frac{8 \lambda^{2}}{1-\alpha} N_{a b},
\end{array}\right.
$$

where $\alpha$ is a non determined proportionality constant. These conditions turn out to leave some freedom in the choice of $G$ and $H$, and therefore in the topology of the spacetime, but are quite restrictive from the point of view of the metrics admitted, as we are going to show below.

The first condition is group-theoretical. It says that the projection on $\mathbf{h}$ of the Killing form of $G$ must be proportional to that of $H$ itself. This restricts the set of possible groups $G$ and $H$. The geometrical meaning is the following. Using the Jacobi identity with respect to the triple of indexes $(i, j, a)$ and the commutation relations eq.(5.1) one finds that the matrices $\left(M_{i}\right)^{a}{ }_{b}:=f_{i b}^{a}$ are a representation of the algebra $\mathbf{h}$ :

$$
\left[M_{i}, M_{j}\right]=f_{i j}^{k} M_{k}
$$

From eq.(5.36) it is seen that the difference between the Killing form (restricted on $\mathbf{h}$ ) $K_{i j}$ and the Killing form of $H K_{i j}^{H}$ is given exactly by the trace of the representation of eq.(5.38). What we are asking is that this trace, which by definition is the Killing form of the representation, is proportional to $K_{i j}^{H}$ itself which is the Killing form of the adjoint representation. This is guaranteed if $H$ is simple, in which case its Lie algebra is irreducible, and therefore the Killing forms of all representations come out to be proportional to each other. If $H$ is semi-simple instead the argument may fail since the adjoint representation is faithful but reducible, and therefore in order for the condition to be satisfied one has to carefully pick up the coefficients $f_{i b}^{a}$ so that they define a representation which is not irreducible but made up of the same direct sum of representations as the adjoint one.

To summarize, the condition is certainly satifisfied by simple groups $H$ like in the case of the $D$-dimensional sphere $S^{D}=S O(D+1) / S O(D)$, the hyperbolic plane $H^{D}=$ $S O(1, D) / S O(D)$ and their Lorentzian analogues de Sitter space $d S^{D}=S O(1, D) / S O(1, D-$ 1 ) and anti-de Sitter $A d S^{D}=S O(2, D-1) / S O(1, D-1)$. The same argument holds if one orbifolds such manifolds by taking the quotient with a finite group $\mathbb{Z}_{n}$, since condition (5.37) only affects the continuous (Lie group) part of the algebra. Therefore real projective spaces $\mathbb{P}^{D}(\mathbb{R})=S^{D} / \mathbb{Z}_{2}$ (which are spin manifolds for $D$ odd) and all further quotients $S^{D} / \mathbb{Z}_{n}, H^{D} / \mathbb{Z}_{n}$ and their Lorentzian analogues apply to our discussion. A counterexample where $H$ is not simple but just semi-simple and (5.35) is still satisfied is given by complex 
projective spaces $\mathbb{C P}^{n}=U(n+1) / U(n) \times U(1)$ (excluding the case $n$ even for which the manifolds do not admit a spin structure).

The second condition concerns the metric of the space. This has to be proportional to the projection of the Killing form on $\mathbf{m}$ indices and, once the constant $\alpha$ is known, different constants of proportionality will lead to different values of the constant $\lambda$ appearing in the Killing equation. The standard metric on the spaces discussed above satisfies the condition, but for example the Fubini-Study metric on $\mathbb{C P}^{n}, n>1$, does not. Hence, once the topology if fixed by the $G / H$ structure, only the standard metric on the manifold can be used.

At this point the reader may be confused by the following fact. Even if we restrict our attention to the simplest case of $\lambda$ real and Riemannian manifolds, it seems that, as long as we find an appropriate quotient $G / H$ satisfying condition (5.37), then we can put the standard metric on it and get a manifold with the maximum number of Killing spinors! This seems to disagree with the fact one would expect only the $D$-dimensional sphere to admit maximum number of Killing spinors. The solution to this apparent puzzle, as mentioned in 4 , is that maximum number of Killing spinors is associated to a flat cone over the manifold, and for this to happen only maximal symmetry is necessary. For example, real and complex projective spaces are maximally symmetric and the former is not even simply connected. Alternatively, using an orbifold $S^{D} / \mathbb{Z}_{n}$ one discover that its cone is a proper cone (in the literal sense), i.e. a manifold with $\mathbb{Z}_{n}$ holonomy which is everywhere flat but on its tip, where the Riemann tensor picks up a $\delta$-like singularity. Not by chance the tip of the cone is set at $r=0$, where $r$ is the radial coordinate of the cone. One can also take the cone over a complex projective space $\mathbb{C P}_{n}$ and still get a space which is everywhere flat but on the origin. The only requirement one has to satisfy in order to get a cone which is flat out of the origin is that the base manifold has, locally, the metric of a maximally symmetric space.

As a final remark we comment on another, different case which is not contemplated in this approach: homogeneous pp-wave spacetimes. They are symmetric spacetimes but the Lie algebra of their isometry group contains a central element. Therefore the Killing form is not invertible and another metric $N_{a b}$ has to be used. This implies the representatives of $P_{a}$ generators cannot be taken to be simply Gamma matrices. As a matter of fact Killing spinors on these spacetimes are not present in the maximum number and therefore cannot be obtained using the procedure outlined in this section. Rather, it can happen instead that, when the pp-wave spacetime is a maximally supersymmetric solution of supergravity, then it is a generalized kind of Killing spinors that appears in maximal number. These correspond to spinors associated to transformations of supergravity fields in presence of fluxes, and accordingly to this the representatives of $P_{a}$ generators are not given by eq. (5.20), but a different definition where the flux appears (see 27] for an example with $\mathcal{N}=1$ in $D=11$ dimensions). The presence of fluxes can give rise to new kind of special tensors on these manifolds, like calibrations, and we reserve their study to future communications. Yano tensors of rank 2 and 3 on a generalized pp-wave background have been studied in [36]. 


\section{Conclusions}

The main purpose of this paper was to study in detail the quantum mechanical role of Yano tensors. We started from the theory of the (quantized) spinning particle and from there have shown that they allow to define operators which commute with the Dirac operator in curved spacetime, for every possible rank of the tensor used. These operators provide quantum numbers for the Dirac equation even when isometries are completely absent. One possible application of this is the study of the Dirac equation on nearly Calabi-Yau or $G_{2}$ manifolds, since they have almost special holonomy. Being linear in the momenta, the operators define genuine dynamical symmetries of the system, i.e. symmetries of the phase space, and could be relevant in the definition of representations of the quantum group of dynamical symmetries on a given spacetime.

Manifolds of special holonomy in general admit Yano and conformal Yano tensors. We have constructed them explicitly for maximally symmetric spaces, showing that their form is extremely simple.

We believe that Yano operators deserve further attention. They are related to a mulititude of different topics like exotic supersymmetries, classes of manifolds which are more general than Kähler ones, index theorems, supergravity theories.

\section{Acknowledgments}

I am grateful to Gary Gibbons for drawing my attention to Yano tensors and for illuminating discussions. Many thanks to Sean Hartnoll, Sigbjorn Hervik, Jasbir Nagi, Carlos Nuñez, Ruben Portugues, Guillermo Silva for useful suggestions along different stages of this work, and for cups of coffee on the roof of CMS. The author is funded by EPSRC, Fondazione "A. Della Riccia", Firenze, and Fondazione "Ing. A. Gini", Padova.

\section{Appendix 1: Proof of the Lemma}

The (anti)commutator splits into a classical and a quantum part. The classical part vanishes due to the result of [6] and [7]. So we just need to check that quantum corrections vanish.

There are two sources of "anomaly" in the quantum corrections. One is related to the fact that the Clifford algebra of Gamma matrices is different from the Grassmann algebra of spin coordinates $\psi^{\mu}$, eq.(2.30). The second is the well known operator ordering problem due to the quantization procedure $\Pi_{\mu} \rightarrow \hbar D_{\mu}$. Direct inspection shows that contributions due to operator ordering are of the same kind of those coming from Gamma matrix (anti)commutators (their only source is given by commutators of covariant derivatives). One can reorganize the expression $\left[\not D, Y_{r}\right\}$ in terms of powers of Gamma matrices. Order by order they are given by $\Gamma^{r+2}, \Gamma^{r}, \Gamma^{r-2}, \ldots$ The term proportional to $\Gamma^{r+2}$ has no contractions since is the one of highest order, therefore is a classical term. Possible terms coming from quantum corrections are those of order $r, r-2, \ldots$ One can easily see that the only terms allowed were contractions appear are the following. 
For order $r$ :

$$
\begin{gathered}
\Gamma^{a_{1} \ldots a_{r}} f_{\left[a_{1} \ldots a_{r-2}\right.}^{b_{1} b_{2}} R_{\left.a_{r-1} a_{r}\right] b_{1} b_{2}}, \\
\Gamma^{a_{1} \ldots a_{r}} f_{\left[a_{1} \ldots a_{r-1}\right.}^{b}(R i c)_{\left.a_{r}\right] b} .
\end{gathered}
$$

For order $r-2$ :

$$
\begin{aligned}
& \Gamma^{a_{1} \ldots a_{r-2}} f_{\left[a_{1} \ldots a_{r-3}\right.}^{b_{1} b_{2} b_{3}} R_{\left.a_{r-2}\right] b_{1} b_{2} b_{3}}=0, \\
& \Gamma^{a_{1} \ldots a_{r-2}} f_{a_{1} \ldots a_{r-2}} b_{1} b_{2}(R i c)_{b_{1} b_{2}}=0 .
\end{aligned}
$$

All orders from $r-2$ downwards vanish because there are no further nonzero contractions. The term $f_{a_{1} \ldots a_{r}} R$ cannot appear since is of order $r$ because $R$ comes from the Riemann tensor after two contractions ${ }^{2}$.

Therefore all that one has to do is to check the order $r$. A detailed calculation shows that there appear two non trivial possible quantum corrections in the (anti)commutator. The first is

$$
\frac{(-1)^{r-1}}{4} \Gamma^{\mu_{1} \ldots \mu_{r}} f_{\left[\mu_{1} \ldots \mu_{r-1}\right.}{ }^{b} R_{\left.\mu_{r}\right] b a_{1} a_{2}} \Gamma^{a_{1} a_{2}}
$$

Using the expansion

$$
\Gamma_{\mu_{1} \ldots \mu_{r}} \Gamma^{a_{1} a_{2}}=\Gamma_{\mu_{1} \ldots \mu_{r}}^{a_{1} a_{2}}+2 \hbar r \delta_{\left[\mu_{1}\right.}^{\left[a_{1}\right.} \Gamma_{\left.\mu_{2} \ldots \mu_{r}\right]}^{\left.a_{2}\right]}+\text { (lower orders) }
$$

one can extract the order $r$ term and show it amounts to

$$
(-1)^{r+1}\left(-\frac{r-1}{4} \Gamma^{a_{1} \ldots a_{r}} f_{\left[a_{1} \ldots a_{r-2}\right.}^{b_{1} b_{2}} R_{\left.a_{r-1} a_{r}\right] b_{1} b_{2}}+\frac{1}{2} \Gamma^{a_{1} \ldots a_{r}} f_{\left[a_{1} \ldots a_{r-1}\right.}{ }^{b}(R i c)_{\left.a_{r}\right] b}\right) .
$$

The second non trivial part is given by

$$
\frac{(-1)^{r+1}}{2(r+1)} \Gamma^{\lambda} \Gamma^{\mu_{1} \ldots \mu_{r+1}} \nabla_{\lambda} \nabla_{\mu_{1}} f_{\mu_{2} \ldots \mu_{r+1}} .
$$

Using the integrability condition (2.21) and the identity

$$
\Gamma^{\lambda} \Gamma_{\mu_{1} \ldots \mu_{r+1}}=\Gamma_{\mu_{1} \ldots \mu_{r+1}}^{\lambda}+\hbar(r+1) \delta_{\left[\mu_{1}\right.}^{\lambda} \Gamma_{\left.\mu_{2} \ldots \mu_{r+1}\right]}
$$

one can calculate the order $r$ contribution and see that it exactly cancels the other term of eq. (6.3). $\triangle$

\footnotetext{
${ }^{2}$ Here we are talking about two contractions appearing algebraically from Gamma matrices commutators. Of course the term $f_{a_{1} \ldots a_{r}} R$ can appear from a term of order $r$ when one contraction is provided from the Riemann tensor rather than an anticommutator of Gamma matrices. For example, on Einstein spaces, it will come from the term $f_{a_{1} \ldots a_{r-1}}{ }^{b}(\text { Ric })_{a_{r} b}$. Therefore we consider this as special case of the discussion of order $r$ terms.
} 


\section{Appendix 2: Hodge duality and conformal Yano equation}

First of all decompose the covariant derivative of a rank $r$ form $f$ according to

$$
\begin{aligned}
& \nabla_{\mu_{1}} f_{\mu_{2} \mu_{3} \ldots \mu_{r+1}}=\nabla_{\left[\mu_{1}\right.} f_{\left.\mu_{2} \mu_{3} \ldots \mu_{r+1}\right]}+ \\
& \frac{2}{r+1}\left(\nabla_{\left(\mu_{1}\right.} f_{\left.\mu_{2}\right) \mu_{3} \ldots \mu_{r+1}}+\nabla_{\left(\mu_{1}\right.} f_{\left.\left|\mu_{2}\right| \mu_{3}\right) \ldots \mu_{r+1}}+\ldots+\nabla_{\left(\mu_{1}\right.} f_{\left.\left|\mu_{2} \mu_{3} \ldots \mu_{r}\right| \mu_{r+1}\right)}\right) .
\end{aligned}
$$

Let $f^{*}$ be the dual of $f$. Then the antisymmetric part of the covariant derivative can be written as

$$
\nabla_{\left[\mu_{1}\right.} f_{\left.\mu_{2} \mu_{3} \ldots \mu_{r+1}\right]}=\frac{(-1)^{D(r+1)+t}}{r+1}\left(* \delta f^{*}\right)_{\mu_{1} \ldots \mu_{r+1}}
$$

using $* *=(-1)^{r(D-r)+t}, \delta=(-1)^{D(r+1)+1+t} * d *$, where $t$ is the number of time dimensions. Suppose now $t=0$ for simplicity. The discussion for $t=1$ is analogous. One can calculate $\nabla f^{*}$ using the decomposition (6.6), obtaining

$$
\begin{aligned}
\nabla_{\mu_{1}} f_{\mu_{2} \mu_{3} \ldots \mu_{(D-r+1)}}^{*}=\frac{(-1)^{r(D-r)}}{r !} \eta_{\mu_{2} \mu_{3} \ldots \mu_{(D-r+1)}} \lambda_{1} \ldots \lambda_{r}[ & \nabla_{\left[\mu_{1}\right.} f_{\left.\lambda_{1} \ldots \lambda_{r}\right]}+ \\
& \left.\frac{2}{r+1}\left(\nabla_{\left(\mu_{1}\right.} f_{\left.\lambda_{1}\right) \ldots \lambda_{r}}+\ldots\right)\right] .
\end{aligned}
$$

Now, the contribution to the totally antisymmetric part of $\nabla f^{*}$ comes only from the terms in round brackets. Considering only this part, and substituting eq.(3.5) in it, we get

$$
\begin{aligned}
& 2 \frac{(-1)^{r(D-r)}}{(r+1) !} \eta_{\mu_{2} \mu_{3} \ldots \mu_{(D-r+1)}}{ }^{\lambda_{1} \ldots \lambda_{r}}\left[g_{\mu_{1} \lambda_{1}} \Phi_{\lambda_{2} \ldots \lambda_{r}}+A g_{\lambda_{2}\left(\mu_{1}\right.} \Phi_{\left.\lambda_{1}\right) \lambda_{3} \ldots \lambda_{r}}\right] \\
= & \frac{r(2-A)}{(r+1) !}(-1)^{r(D-r)} \eta_{\mu_{2} \mu_{3} \ldots \mu_{(D-r+1)} \mu_{1}}^{\lambda_{2} \ldots \lambda_{r}} \Phi_{\lambda_{2} \lambda_{3} \ldots \lambda_{r}} \\
= & (-1)^{r+1} \frac{2-A}{r+1} * \Phi_{\mu_{1} \ldots \mu_{(D-r+1)}}=(-1)^{r} \frac{2-A}{(D+A)(r+1)} * \delta f_{\mu_{1} \ldots \mu_{(D-r+1)}} .
\end{aligned}
$$

On the other hand, substituting $f$ with $f^{*}$ in (6.7) gives

$$
\begin{aligned}
\nabla_{\left[\mu_{1}\right.} f_{\left.\mu_{2} \mu_{3} \ldots \mu_{D-r+1}\right]}^{*} & =\frac{(-1)^{D((D-r)+1)}+t}{(D-r)+1}\left(* \delta * f^{*}\right)_{\mu_{1} \ldots \mu_{D-r+1}} \\
& =\frac{(-1)^{r}}{D-r+1}(* \delta f)_{\mu_{1} \ldots \mu_{D-r+1}},
\end{aligned}
$$

which is consistent with the previous expression if and only if $A=1-r$. Once $A$ is fixed a complete expression of $\nabla f^{*}$ can be calculated from (6.8) and the result is:

$$
\nabla_{\mu_{1}} f_{\mu_{2} \mu_{3} \ldots \mu_{D-r+1}}^{*}=\nabla_{\left[\mu_{1}\right.} f_{\left.\mu_{2} \mu_{3} \ldots \mu_{D-r+1}\right]}^{*}-\frac{D-r}{r+1} g_{\mu_{1}\left[\mu_{2}\right.} \delta f_{\left.\mu_{3} \ldots \mu_{D-r+1}\right]}^{*},
$$

which implies

$$
\nabla_{\left(\mu_{1}\right.} f_{\left.\mu_{2}\right) \mu_{3} \ldots \mu_{D-r+1}}^{*}=-\frac{1}{r+1}\left(g_{\mu_{1} \mu_{2}} \delta f_{\mu_{3} \ldots \mu_{D-r+1}}^{*}-(D-r-1) g_{\left[\mu_{3} \mid\left(\mu_{1}\right.\right.} \delta f_{\left.\mu_{2}\right) \mid \mu_{4} \ldots \mu_{D-r+1}}^{*}\right),
$$

that is in agreement with the conformal Yano equation (3.6) and the condition $\Phi_{f^{*}}=-\frac{1}{r+1} \delta f^{*}$. When the signature is Lorentzian the only difference is the appearence of some extra $(-1)$ terms.

From what shown above we can easily see that the dual of a Yano tensor $f$ is a closed conformal Yano tensor, and in particular it is a Yano tensor itself if and only if both $f$ and $f^{*}$ are harmonic. 


\section{Appendix 3: Some examples of spaces of (almost) special holonomy}

In this section we give some example of spaces with special or almost special $S U(3)$ and $G_{2}$ holonomy, to which our construction applies. They are either $G_{2}$ holonomy spaces which are asymptotically locally conical (ALC) spaces over manifolds with almost $S U(3)$ holonomy, or Spin(7) spaces which are ALC over manifolds with almost $G_{2}$ holonomy. Such spaces are of direct relevance in compactifications of String/ $M$-Theory that preserve either $\mathcal{N}=1$ or $\mathcal{N}=2$ supersymmetry, with or without fluxes. We refer in the following to 34 and 35].

\section{$G_{2}$ holonomy metrics}

Spaces of $G_{2}$ holonomy can arise in compactifying $M$-Theory down to four dimensions while preserving $\mathcal{N}=1$ supersymmetry. It is well known that compactification on a smooth $G_{2}$ manifold cannot lead to chiral fermions (37]), so that an alternative approach is to use singular $G_{2}$ manifolds. The metrics we present here have $G_{2}$ holonomy and are locally asymptotic to conical metrics. From the metric on the cone one can immediately read the metric on the base manifold, which will be automatically of almost $S U(3)$ holonomy. In [38] is shown how to associate, to every such non-compact ALC manifold of $G_{2}$ holonomy, a compact manifold with almost $G_{2}$ holonomy and conical singularities. This means that the latter, close to the conical singularities, looks like the former ALC manifold. Studying the Dirac equation on such compact manifolds using conserved quantities generated by Yano tensors may be directly relevant in establishing the existence of chiral fermions, as an approach that is complementary to those already used in the literature [39]. In all these cases the possible antisymmetric tensors that can be defined by (4.14), (4.15) and are non zero are given by a 3 -form $\phi$ and its dual $* \phi$. Since they are covariantly constant, each of these is both a calibration for the $G_{2}$ manifold and a Yano tensor. It would be interesting to see whether these forms, as well as in the rank 2 case, can provide genuine String Theory symmetries. In the conical limit one recovers a metric of almost $S U(3)$ holonomy on the base of the cone. Then the forms that are present on the base manifold are a dimensional reduction of $\phi, * \phi$, and are an almost complex structure $J$, a holomorphic volume form $\rho$ and its dual $* \rho$. They satisfy the conditions $J_{\lambda \mu} J^{\mu}{ }_{\nu}=-g_{\lambda \nu}, d J \sim \rho, d * \rho \sim J J$. $J$ is a Yano tensor of kind $A$, while $* \rho$ a Yano tensor of kind $B$ and $\rho$ a conformal Yano tensor (see 40] for a discussion of almost $S U(3)$ holonomy).

Hence, the link between Yano tensors, special holonomy, supergravity and calibrations is manifest.

$G_{2}$ metrics over $S U(3) / U(1) \times U(1)$.

Let $L_{A}{ }^{B}, A, B=1,2,3$, be left invariant forms of $S U(3), L_{A}{ }^{A}=0,\left(L_{A}{ }^{B}\right)^{\dagger}=L_{B}{ }^{A}$. Left invariant forms for $S U(3) / U(1) \times U(1)$ are given by $\sigma=L_{1}{ }^{3}, \Sigma=L_{2}{ }^{3}, \nu=L_{1}{ }^{2}$. They split into real forms defined by $\sigma=\sigma_{1}+i \sigma_{2}$, etc. Then the following metric has $G_{2}$ holonomy 
and principal orbits given by $S U(3) / U(1) \times U(1)$ :

$$
\begin{aligned}
& d s_{7}^{2}=\frac{d r^{2}}{u v w}+\frac{u w}{v}\left(\sigma_{1}^{2}+\sigma_{2}^{2}\right)+\frac{u v}{w}\left(\Sigma_{1}^{2}+\Sigma_{2}^{2}\right)+\frac{v w}{u}\left(\nu_{1}^{2}+\nu_{2}^{2}\right), \\
& u^{2}=4\left(r-r_{1}\right), \quad v^{2}=4\left(r-r_{2}\right), \quad w^{2}=4\left(r-r_{3}\right),
\end{aligned}
$$

where $r_{1}, r_{2}, r_{3}$ are constants of integration. The metric is singular unless two of the $r_{i}$ are equal. The asymptotical behaviour at $r \rightarrow+\infty$ is given by

$$
d s_{7}^{2}=d R^{2}+R^{2}\left(\sigma_{1}^{2}+\sigma_{2}^{2}+\Sigma_{1}^{2}+\Sigma_{2}^{2}+\nu_{1}^{2}+\nu_{2}^{2}\right),
$$

where $R=\sqrt{2} r^{1 / 4}$. Then one obtains a metric of almost $S U(3)$ holonomy on the base. The rank 3 Yano tensor is given by

$$
\phi=\sqrt{u v w} \operatorname{Re}(\bar{\sigma} \Sigma \nu)+i\left(-\frac{u w}{v} \sigma \bar{\sigma}+\frac{u v}{w} \Sigma \bar{\Sigma}+\frac{v w}{u} \nu \bar{\nu}\right) d t
$$

where $d r=\sqrt{u v w} d t$.

\section{$G_{2}$ metrics over $\mathbb{C P}^{3}$.}

Take $L_{A B}, L_{A B}=-L_{B A}, A=0,1, \ldots 4$, to be left invariant forms of $S O(5)$. Split the index $A$ into $A=(a, 4), a=0, \ldots 3$, and define $P_{a}:=L_{a 4}, R_{i}:=\frac{1}{2}\left(L_{0 i}+\frac{1}{2} \epsilon_{i j k} L_{j k}\right)$, $L_{i}:=\frac{1}{2}\left(L_{0 i}-\frac{1}{2} \epsilon_{i j k} L_{j k}\right), i=1,2,3$. Then one can write

$$
\mathbb{C P}^{3}=\frac{S O(5)}{S U(2)_{L} \times U(1)_{R}},
$$

where $S U(2)_{L}$ is generated by the forms $L_{i}$, and $U(1)_{R}$ by $R_{3}$. The following metric has $G_{2}$ holonomy and $\mathbb{C P}^{3}$ principal orbits:

$$
d s_{7}^{2}=\left(1-\frac{l^{4}}{r^{4}}\right)^{-1} d r^{2}+r^{2}\left(1-\frac{l^{4}}{r^{4}}\right)\left(R_{1}^{2}+R_{2}^{2}\right)+\frac{r^{2}}{2} P_{a}^{2},
$$

and $l$ is a constant. The conical limit is evident. The Yano tensor $\phi$ can be written as

$$
\begin{aligned}
\phi= & d t\left[a^{2} R_{1} R_{2}-b^{2}\left(P_{0} P_{3}+P_{1} P_{2}\right)\right] \\
& +a b^{2}\left[R_{2}\left(P_{0} P_{1}+P_{2} P_{3}\right)-R_{1}\left(P_{0} P_{2}+P_{3} P_{1}\right)\right],
\end{aligned}
$$

where $d r=\sqrt{1-l^{4} / r^{4}} d t$ and the functions $a(t), b(t)$ obey the first order equations $\dot{a}=$ $\frac{1}{2} a^{2} b^{-2}$ and $\dot{b}=-\frac{1}{2} a b^{-1}$.

$G_{2}$ metrics over $S^{3} \times S^{3}$.

This time the base manifold will be $S^{3} \times S^{3}=S U(2) \times S U(2) / \mathbb{Z}_{2}$. Let $\sigma_{i}, \Sigma_{i}, i=1,2,3$, left-invariant forms associated to the two copies of $S U(2)$. A metric with $G_{2}$ holonomy is given by

$$
d s_{7}^{2}=d r^{2}+a_{i}^{2}(r)\left(\sigma_{i}-\Sigma_{i}\right)^{2}+b_{i}^{2}(r)\left(\sigma_{i}+\Sigma_{i}\right)^{2}
$$


$\mathbb{Z}_{2}$ symmetry is associated to the exchange of the two copies of $S U(2)$. The functions $a_{i}, b_{i}$ have to satisfy a set of differential equations explicitly given in [34, pg.46. For what concerns the behaviour at infinity, a subset of all possible solutions is given by those satisfying $a_{2}=a_{3}, b_{2}=b_{3}$. These are all regular solutions and at $r \rightarrow+\infty$ behave as

$$
d s_{7}^{2} \sim d r^{2}+\frac{r^{2}}{4}\left(\left(\sigma_{1}-\Sigma_{1}\right)^{2}+\frac{3}{4}\left(\sigma_{2}-\Sigma_{2}\right)^{2}+\frac{3}{4}\left(\sigma_{2}+\Sigma_{2}\right)^{2}\right) .
$$

The Yano tensor for the general metric is

$$
\begin{aligned}
\phi & =a_{1} a_{2} a_{3}\left(\sigma_{1}-\Sigma_{1}\right)\left(\left(\sigma_{2}-\Sigma_{2}\right)\left(\sigma_{3}-\Sigma_{3}\right)-a_{1} b_{2} b_{3}\left(\sigma_{1}-\Sigma_{1}\right)\left(\left(\sigma_{2}+\Sigma_{2}\right)\left(\sigma_{3}+\Sigma_{3}\right)\right.\right. \\
& +a_{2} b_{1} b_{3}\left(\sigma_{2}-\Sigma_{2}\right)\left(\left(\sigma_{1}+\Sigma_{1}\right)\left(\sigma_{3}+\Sigma_{3}\right)-a_{3} b_{1} b_{2}\left(\sigma_{3}-\Sigma_{3}\right)\left(\left(\sigma_{1}+\Sigma_{1}\right)\left(\sigma_{2}+\Sigma_{2}\right)\right.\right. \\
& +d r a_{i} b_{i} \sigma_{i} \Sigma_{i} .
\end{aligned}
$$

\section{$\operatorname{Spin}(7)$ holonomy metrics}

Here we contemplate two cases of spaces with Spin(7) holonomy. Almost Spin(7) holonomy does not exist since there are no possible cones of dimension 9 that admit covariantly constant spinors. In the asymptotically conical limit one recovers almost $G_{2}$ metrics on the base manifolds. The only non zero $p$-form is a self-dual 4 -form $\Omega$, which is both a calibration and a Yano tensor. On the base of the cone, by dimensional reduction and consistently with almost $G_{2}$ holonomy, one recovers a 3 -form $\phi$ and a 4 -form $* \phi$. $\phi$ is a type $B$ Yano tensor that satisfies $d \phi \sim * \phi$, and is a calibration, while $* \phi$ is type $B$ conformal Yano tensor such that $d * \phi=0$. The common feature of all the cases discussed so far is that calibrations are always Yano tensors, whether flux is or is not turned on.

$\operatorname{Spin}(7)$ metrics over $S O(5) / S U(2)$.

Here $S O(5) / S U(2)_{L}=S^{7}$ is defined in the same way as in the case of $G_{2}$ metrics over $\mathbb{C P}^{3}$, but without taking the quotient with respect to the $U(1)_{R}$ generator $R_{3}$. A metric with $\operatorname{Spin}(7)$ holonomy is given by

$$
d s_{8}^{2}=d r^{2}+a_{i}(r)^{2} R_{i}^{2}+b(r)^{2} P_{a}^{2},
$$

where the functions $a_{i}, b$ satisfy the differential equations

$$
\begin{aligned}
& \dot{a}_{1}=\frac{a_{1}^{2}-\left(a_{2}-a_{3}\right)^{2}}{a_{2} a_{3}}-\frac{a_{1}^{2}}{2 b^{2}}, \quad \text { and cyclic, } \\
& \dot{b}=\frac{1}{4} \sum_{i=1}^{3} \frac{a_{i}}{b} .
\end{aligned}
$$

One particular solution is given by

$$
a_{i}=-\frac{\lambda}{4} r A_{i}, \quad b=-\frac{\lambda}{4} r B,
$$

with

$$
\begin{aligned}
& \lambda A_{1}=-4 \frac{A_{1}^{2}-\left(A_{2}-A_{3}\right)^{2}}{A_{2} A_{3}}+2 \frac{A_{1}^{2}}{B^{2}}, \quad \text { and cyclic, } \\
& \lambda B^{2}=-\sum_{i=1}^{3} A_{i},
\end{aligned}
$$


and it is particularly easy to see the conical limit in this case. Since it is of cohomogeneity one, it is easy to display a rank four Yano tensor: it is constructed from the vielbein $e^{A}$ as

$$
\Omega=\frac{1}{4 !} \Psi_{A B C D} e^{A} e^{B} e^{C} e^{D},
$$

where $\Psi_{A B C D}$ is a $\operatorname{Spin}(7)$ tensor constructed as following:

$$
\begin{aligned}
& \Psi_{a b c 8}=\psi_{a b c}, \\
& \Psi_{a b c d}=\epsilon_{m_{1} m_{2} m_{3} a b c d} \psi_{m_{1} m_{2} m_{3}},
\end{aligned}
$$

where the index $a, b$ ranges from 1 to 7 and $\psi_{a b c}$ is the $G_{2}$ invariant antisymmetric tensor whose non zero components are given by

$$
\psi_{123}=\psi_{516}=\psi_{624}=\psi_{435}=\psi_{471}=\psi_{673}=\psi_{572}=1 .
$$

$\Omega$ decomposes over the almost $G_{2}$ base as

$$
\Omega=\left(\frac{\lambda r}{4}\right)^{3} d r \phi+\left(\frac{\lambda r}{4}\right)^{4} * \phi,
$$

such that

$$
\tilde{d} \phi=\lambda * \phi .
$$

, where $\tilde{d}$ is the differential operator on the base.

\section{$\operatorname{Spin}(7)$ metrics over $S U(3) / U(1)$.}

In this case principal orbits are given by Aloff-Wallach spaces. These are spaces of the form $S U(3) / U(1)$, where $U(1)=\operatorname{diag}\left(e^{i k}, e^{i l}, e^{-i(k+l)}\right)$. When $k, l$ are relatively prime the spaces are simply connected and denoted by $N(k, l)$. Almost $G_{2}$ metrics on these spaces are less interesting since they are smooth, without singularities. Take $S U(3) / U(1) \times U(1)$ generators as in the above example of $G_{2}$ metrics over the space, and add the generator $\lambda=\sqrt{2} \cos \tilde{\delta} L_{1}{ }^{1}+\sqrt{2} \sin \tilde{\delta} L_{2}{ }^{2}$, with $\tan \tilde{\delta}=-k / l$. Then $\operatorname{Spin}(7)$ metrics are given by

$$
d s_{8}^{2}=d r^{2}+a(r)^{2} \sigma_{i}^{2}+b(r)^{2} \Sigma_{i}^{2}+c(r)^{2} \nu_{i}^{2}+f^{2} \lambda^{2},
$$

where the functions $a, b, c, f$ satisfy the differential equations

$$
\begin{aligned}
\dot{a} & =\frac{b^{2}+c^{2}-a^{2}}{b c}-\frac{\sqrt{2} f \cos \tilde{\delta}}{a}, \\
\dot{b} & =\frac{a^{2}+c^{2}-b^{2}}{c a}+\frac{\sqrt{2} f \sin \tilde{\delta}}{b}, \\
\dot{c} & =\frac{a^{2}+b^{2}-c^{2}}{a b}+\frac{\sqrt{2} f(\cos \tilde{\delta}-\sin \tilde{\delta})}{c}, \\
\dot{f} & =\frac{\sqrt{2} f^{2}(\cos \tilde{\delta}-\sin \tilde{\delta})}{c^{2}}+\frac{\sqrt{2} f^{2} \cos \tilde{\delta}}{a^{2}}-\frac{\sqrt{2} f^{2} \sin \tilde{\delta}}{b^{2}} .
\end{aligned}
$$

One particular solution is given by

$$
a=A r, b=B r, c=C r, f=F r,
$$

with

$$
\begin{aligned}
& \frac{l F}{\mu A^{2}}=\frac{B^{2}+C^{2}-A^{2}}{A B C}-1, \quad \text { and cyclic, } \\
& \left(\frac{l}{A^{2}}+\frac{k}{B^{2}}-\frac{l+k}{C^{2}}\right) \frac{F}{\mu}=1,
\end{aligned}
$$

$\sqrt{2} \mu=\sqrt{k^{2}+l^{2}}$. These equations define an almost $G_{2}$ metric on the base of the cone. 


\section{References}

[1] C.B. Collins, Gen. Rel. Grav. 10 (1979) 925.

[2] K. Yano, Ann. Math. 55 (1952) 328.

[3] R. Floyd, The dynamics of Kerr fields. Ph.D. thesis, London University (1973).

[4] R. Penrose, Ann. N. Y. Acad. Sci. 224, 125 (1973).

[5] B. Carter and R. G. McLenaghan, Phys. Rev. D 19 (1979) 1093.

[6] G. W. Gibbons, R. H. Riedtdijk, J. W. van Holten, Nucl. Phys. B 404 (1993) 42-64.

[7] M. Tanimoto, Nucl. Phys. B 442 (1995) 549-560.

[8] J. A. de Azcárraga, J. M. Izquierdo, A. J. Macfarlane, Nucl. Phys. B 604 (2001) 75-91.

[9] A. J. Macfarlane, Nucl. Phys. B 621 (2002) 712-722.

[10] J.-W. van Holten, K. Peeters and A. Waldron, Class. and Quant. Grav. 16 (1999) 2537, hep-th/9901163.

[11] K. Peeters and A. Waldron, J. High Energy Phys. 02 (1999) 24, hep-th/9901016.

[12] F. A. Berezin and M. S. Marinov, Ann. Phys. (NY) 104 (1977) 336.

[13] R. Casalbuoni, Phys. Lett. B 62 (1976) 49.

[14] A. Barducci and R. Casalbuoni, L. Lusanna, Nuovo Cim. 35A (1976) 377, Nucl. Phys. B $124(1977) 93$.

[15] L. Brink, S. Deser, B. Zumino, P. DiVecchia and P. Howe, Phys. Lett. B 64 (1976) 43.

[16] L. Brink, P. DiVecchia and P. Howe, Nucl. Phys. B 118 (1977) 76.

[17] J. W. van Holten, in Proc. Sem. Math. structures in field theories, 1986-87, CWI syllabus Vol.26 (1990), p.109.

[18] J. W. van Holten and R. H. Rietdijk, Class. and Quant. Grav. 7 (1990) 247.

[19] J. W. van Holten and R. H. Rietdijk, hep-th/9205074; R. H. Rietdijk, Applications of supersymmetric quantum mechanics, Ph.D. Thesis, Amsterdam (1992).

[20] R. H. Riedtdijk, J. W. van Holten, Nucl. Phys. B 472 (1996) 427-446.

[21] D. Baleanu, S. Baskal, Int. J. Mod. Phys. A 17 (2002) 3737-3748.

[22] S. Tachibana, Tohoku Math. J. 21 (1969) 56.

[23] E. N. Glass and J. Kress, gr-qc/9809074.

[24] J. J. Ferrando and J. A. Saez, gr-qc/0212085.

[25] S. E. Stepanov, Theor. Math. Phys. 134(3) (2003) 333-338.

[26] D. D. Joyce. Compact Manifolds with Special Holonomy. Oxford University Press, 2000, pg.66.

[27] N. Alonso-Alberca, E. Lozano-Tellechea and T. Ortín, hep-th/0208158.

[28] C. Bär, Commun. Math. Phys. 154 (1993) 509-521.

[29] S. Gallot, Ann. Sci. École Norm. Sup. 12 (1979) 235-267. 
[30] J. M. Figueroa-O'Farrill, hep-th/9902066.

[31] H. Baum, T. Friedrich, R. Grunewald, I. Kath. Twistors and Killing spinors on Riemannian manifolds. Sektion Mathematik der Humboldt-Universität zu Berlin, 1989, 179 S.

[32] F. Leitner, math.DG/0302024.

[33] F. De Jonghe, K. Peeters, K. Sfetsos, Class. and Quant. Grav. 14 (1997) 35-46, hep-th/9607203.

[34] M. Cvetič, G. W. Gibbons, H. Lü and C. N. Pope, hep-th/0108245.

[35] A. Bilal, J. P. Derendinger and K. Sfetsos, hep-th/0111274.

[36] D. Baleanu and S. Baskal, gr-qc/0206045.

[37] E. Witten, Nucl. Phys. B 186 (1981) 412.

[38] A. Bilal and S. Metzger, hep-th/0302021.

[39] S. A. Hartnoll, Phys. Lett. B 532 (2002) 297-304.

[40] M. Cvetič, G. W. Gibbons, H. Lü and C. N. Pope, hep-th/0206151. 